\title{
A feedback control model of immunogenic tumours with comprehensive therapy
}

\author{
Biao Tang \\ Department of Applied Mathematics, Xi'an Jiaotong University, \\ $X i$ 'an 710049, PR China \\ tang3112054020@stu.xjtu.edu.cn \\ Yanni Xiao \\ Department of Applied Mathematics, Xi'an Jiaotong University, \\ Xi'an 710049, PR China \\ yxiao@mail.xjtu.edu.cn \\ Sanyi Tang \\ College of Mathematics and Information Science, Shaanxi Normal University, \\ Xi'an, 710062, PR China \\ sytang@snnu.edu.cn \\ Robert A. Cheke \\ Natural Resources Institute, University of Greenwich at Medway, \\ Central Avenue, Chatham Maritime, Chatham, Kent, ME4 4 TB, UK \\ r.a.cheke@greenwich.ac.uk \\ Received (to beinserted by publisher)
}

In the traditional therapy of cancers, surgery is a main method but it often fails to cure patients for complex reasons. Thus, a new therapeutic approach including both surgery and immunotherapy has been proposed and shown to be effective clinically in inhibiting cancer cells while retaining immunologic memory. This comprehensive strategy guided by a threshold of tumour cells in an immune tumour system is modelled and conditions for successful control of tumours and related dynamics are addressed. A mathematical model with state-dependent impulsive interventions is formulated to describe surgery combined with immunotherapy. By analyzing the properties of the Poincaré map we examine the global dynamics of the immune tumour system with state-dependent feedback control, including the existence and stability of the semi-trivial order-1 periodic solution and the positive order-k periodic solution. The main results showed that surgery alone can only control the tumour size below a certain level while there is no immunologic memory. If comprehensive therapy involving combining surgery with immunotherapy is considered, then not only can the cancers be controlled below a certain level, but the immune system can also retain its activity. The existence of positive order-k periodic solutions implies that periodical therapy is needed to control the cancers, however choosing the treatment frequency and the strength of the therapy remains challenging, and hence a strategy of individual-based therapy is suggested.

Keywords: Tumour cell; Immunotherapy; Poincaré map; State-dependent feedback control; Order-k periodic solution

*Author for correspondence. 


\section{Introduction}

As is well known cancer, resulting from the progressive growth of the progeny of a single transformed cell, is one of the three leading causes of death in industrialized nations. Surgery is traditionally the main therapy for controlling cancers. However, surgery often fails to cure patients because either micrometastases that were undetectable at the time of surgery grow and cause resurgence of disease or because of local recurrence. In recent years, a new method (i.e. immunotherapy) has been investigated in experiments, as well performed clinically. Immunotherapy is an attractive way to increase cancer cure rates and ultimately lead to effective cancer prevention strategies, as shown by [Gubin et al., 2014; Powles et al., 2014; Schmiegel et al., 1997; Símová et al., 2010; Sunay et al., 2013]. It aims at clearing the tumour cells mainly by enhancing the immune function of patients.

The immune response to a tumour is usually cell-mediated by T-cells such as cytotoxic T lymphocytes. Much attention has been paid to the interactions between the immune system and a growing tumour in [Angelis et al., 2003; De Pillis \& Radunskaya, 2003; Bodbar \& Bodbar, 2003; Karen \& Jonathan, 2005; Lefever et al., 1992; Look et al., 1981; Salleem \& Angrawal, 2012; Subuyanto et al., 2014]. With models such as those described in these papers numerical estimates of biologically significant parameters have been obtained and interesting phenomena interpreted. In [Janeway et al., 2001; Shial ét al., 2011], the authors described various methods of immunotherapy: a) adoptive immunotherapy (AIT): direct injection into patients of effector cells cultured in vitro; b) active immunotherapy including injecting immunomodulators to non-specifically enhance the immune function, and then activate the immunotherapy, or injecting tumour vaccine to induce specific immune responses to a tumour. To date, the dynamics of the immunogenic tumour system with immunotherapy have been discussed by many authors in [Arcietro et al., 2004; Borges et al., 2014; Kuznetsov \& Knott, 2001; Kuznetsov et al., 1994; Wilson \& Levy, 2012]. A simple model of immunogenic tumours with immunotherapy was proposed in [Kuznetsov et al., 1994], and given as the follows:

$$
\left\{\begin{array}{l}
\dot{x}=r x(1-\eta x)-p x y \\
\dot{y}=\frac{c x y}{1+\omega x}-q x y-\delta y
\end{array}\right.
$$

where $x$ and $y$ represent the tumour cells and the effector cells, respectively; $r$ is the growth rate of tumour cells which incorporates both their multiplication and death; $1 / \eta$ denotes the carrying capacity; $\delta$ is the death rate of the effector cells, $p$ denotes the rate of binding of effector cells to tumour cells, $q$ represents the rate of inactivation of effector cells, and $c x /(1+\omega x)$ denotes the rate at which effector cells accumulate due to the presence of a tumour.

A common assumption for the model in the paper [Kuznetsov et al., 1994] is that immunotherapy is applied irrespective of the size of the tumour population. However, strategies with pulsed therapy are the usual practise for treating cancers both in experiments and clinics, with fixed timings for the pulses, as described by [Hegmans et al., 2005; Yamaguchi et al., 2006] or with state-dependent strategies [Duffey et al., 2004; Herring et al., 2001; Steve et al., 2005; Walther et al., 1999]. In the paper [Steve et al., 2005], the authors conducted an experiment in which partial tumour-debulking surgery was done when the average tumour size was around $50 \mathrm{~mm}^{2}$, and then immunotherapy was carried out. They concluded that partial de-bulking followed by combination therapy can lead to a long-term cure, with the cured animals having immunologic memory and protection from re-challenge. Moreover, several researchers [Duffey et al., 2004; Herring et al., 2001; Walther et al., 1999] implemented a similar therapy strategy with surgical resection conducted whenever the diameter of a renal tumour reached $3 \mathrm{~cm}$. Under this regime, a proportion of the patients did not need surgery for renal tumours remaining below $3 \mathrm{~cm}$, while others had periodical surgery several times, but none of the patients developed renal tumour metastases. On the basis of these facts, we clearly know there is a feedback control mechanism for cancer therapy. Here the control signal would be the tumour size. That is, once the tumour size reaches a critical value we will carry out the therapy for tumour, otherwise we will not do any therapy. For a cancer the number of tumour cells per volume are supposed to be almost fixed. Then, there will be a critical value of the tumour population size associated with a certain size, denoted by $V_{L}$, to determine when the comprehensive therapy is carried out. Moreover, the threshold 
level was shown to be essential for governing whether the treatment was successful or not [Duffey et al., 2004; Herring et al., 2001; Walther et al., 1999; Xiao et al., 2013].

However, in clinics, either surgery alone or immunotherapy alone is rarely curative for advanced tumours, and so a good choice is to combine the two methods. To the best of the authors' knowledge, few models have been formulated to investigate how comprehensive therapy including surgery and immunotherapy will affect the tumour system and what the resulting dynamics of the tumour system would be. Moreover, there are many challenges involved in examining how treatment frequency and the intensity of the therapy affect the efficiency of the treatment of cancers. Quantifying these issues in a mathematical modelling framework is the main objective of this study.

The purpose of this study was to examine quantitatively what comprehensive therapy interactions can be used to effectively control the growth of tumour cell populations, and further, to address how these key factors (including the instantaneous rate of resection, a constant injection rate of effector cells and a threshold level) affect the success of tumour control. To do this, a mathematical model with statedependent feedback control measures describing such comprehensive therapy processes is proposed and theoretically analyzed. Based on model (1) and including comprehensive therapy for tumour cells we propose the following feedback control model with a threshold:

$$
\left\{\begin{aligned}
\frac{d x(t)}{d t} & =r x(t)(1-\eta x(t))-p x(t) y(t), \\
\frac{d y(t)}{d t} & =\frac{c x(t) y(t)}{1+\omega x(t)}-q x(t) y(t)-\delta y(t)
\end{aligned}\right\} x<V_{L}
$$

with $x\left(t^{+}\right)=\lim _{s \rightarrow t^{+}} x(s), y\left(t^{+}\right)=\lim _{s \rightarrow t^{+}} y(s)$. Here $\theta$ denotes the instantaneous rate of resection of tumour cells after surgery, $\tau$ represents the constant injection rate of effector cells, and $\rho$ denotes the growth rate of effector cells due to active immunotherapy. From the model (2), we can see that once the tumour size reaches the critical value $V_{L}$, the comprehensive therapy is carried out, which leads to the effector cells increasing to $y\left(t^{+}\right)$from $y(t)$ immediately and the tumour cells decreasing to $x\left(t^{+}\right)$from $x(t)$ right away. Quantitative theory for such impulsive systems has been extensively developed [Bonotto, 2009; Bonotto \& Federson, 2008; Liang et al., 2013; Qian et al., 2010; Zeng et al., 2006] which has applications in many domains of applied science, such as in pest management programmes [Tang \& Cheke, 2005, 2008; Tang et al., 2013], virus dynamical systems [Lou et al., 2012; Yang \& Xiao, 2012; Yang et al., 2013], vaccination strategies and epidemiology [Nie et al., 2013; Shulgin et al., 1998], diabetes mellitus [Tang \& Xiao, 2007], and neuron systems [Touboul \& Brette, 2009].

Our paper is organized as follows. In the next section, we first consider the generalized planar impulsive system and the dynamics of the ODE system. In section 3, we mainly investigate the properties of the Poincaré map which is used throughout this paper. Then the semi-trivial order-1 periodic solution and the positive order-1 periodic solution are discussed when $\tau=0$. In section 4 , we investigate the existence and stability of the positive order-k periodic solution when $\tau>0$. Finally, the biological meaning and concluding remarks are discussed in section 5 .

\section{Preliminaries}

\subsection{Impulsive semi-dynamic system and lemmas}

First, we consider the following generalized planar impulsive semi-dynamic system

$$
\left\{\begin{array}{l}
\frac{d x}{d t}=P(x, y), \frac{d y}{d t}=Q(x, y), \text { if } \Psi(x, y) \neq 0, \\
\triangle x=a(x, y), \triangle y=b(x, y), \text { if } \Psi(x, y)=0,
\end{array}\right.
$$


where $(x, y) \in R^{2}, \triangle x=x^{+}-x$ and $\triangle y=y^{+}-y . P, Q, a, b$ are continuous functions from $R^{2}$ into $R$. The impulsive function $I: R^{2} \rightarrow R^{2}$ is defined as follows:

$$
I(x, y)=\left(I_{1}(x, y), I_{2}(x, y)\right)=(x+a(x, y), y+b(x, y))
$$

and $Z^{+}=\left(x^{+}, y^{+}\right)$is called an impulsive point of $Z=(x, y)$.

Let $\left(R^{2}, \pi\right)$ be a planar semi-dynamic system (see the definition in [Bonotto \& Federson, 2008]). For any $Z \in R^{2}$, the positive orbit of $Z$ is given by $C^{+}(z)=\left\{\pi(Z, t) \mid t \in R^{+}\right\}$which is denoted by $\pi^{+}(Z)$. And we define $F(Z, t)=\left\{Z^{\prime} \mid \pi\left(Z^{\prime}, t\right)=Z\right\}$ for $t \geq 0$ and $Z \in R^{2}$.

Based on the above notation, we can define the impulsive semi-dynamic system (see details in [Bonotto \& Federson, 2008; Kual, 1990]) as follows:

Definition 1. A planar impulsive semi-dynamic system $\left(R^{2}, \pi ; M, I\right)$ consists of a continuous semi-dynamic system $\left(R^{2}, \pi\right)$ together with a nonempty closed subset $M$ of $R^{2}$ and a continuous function $I: M \rightarrow R^{2}$ such that for every $Z \in M$, there exists a $\epsilon_{Z}>0$ such that

$$
F\left(Z,\left(0, \epsilon_{Z}\right)\right) \cap M=\emptyset \quad \text { and } \quad \pi\left(Z,\left(0, \epsilon_{Z}\right)\right) \cap M=\emptyset .
$$

Definition 2. A trajectory $\pi^{+}(Z)$ of $\left(R^{2}, \pi ; M, I\right)$ is said to be order $k$ periodic if there exist nonnegative integers $m$ and $k$ such that $k$ is the smallest integer for which $I^{m}(Z)=I^{m+k}(Z)$ and $Z \in M$.

The following lemmas are useful for determining the stability and bifurcation of the effector cell free periodic solution.

Lemma 1. (Analogue of Poincaré Criterion) The order-k periodic solution $x=\xi(t), y=\zeta(t)$ of system (3) is orbitally asymptotically stable and enjoys the property of asymptotic phase if the multiplier $\mu_{2}$ satisfies the condition $\left|\mu_{2}\right|<1$. Where

$$
\begin{aligned}
& \mu_{2}=\prod_{k=1}^{q} \triangle_{k} \exp \left[\int_{0}^{T}\left(\frac{\partial P}{\partial x}(\xi(t), \zeta(t))+\frac{\partial Q}{\partial y}(\xi(t), \zeta(t))\right]\right. \\
& \triangle_{k}=\frac{P_{+}\left(\frac{\partial b}{\partial y} \frac{\partial \Psi}{\partial x}-\frac{\partial b}{\partial x} \frac{\partial \Psi}{\partial y}+\frac{\partial \Psi}{\partial x}\right)+Q_{+}\left(\frac{\partial a}{\partial x} \frac{\partial \Psi}{\partial y}-\frac{\partial a}{\partial y} \frac{\partial \Psi}{\partial x}+\frac{\partial \Psi}{\partial y}\right)}{P \frac{\partial \Psi}{\partial x}+Q \frac{\partial \Psi}{\partial y}}
\end{aligned}
$$

and $P, Q, \frac{\partial a}{\partial x}, \frac{\partial a}{\partial y}, \frac{\partial b}{\partial x}, \frac{\partial b}{\partial y}, \frac{\partial \Psi}{\partial x}, \frac{\partial \Psi}{\partial y}$ are all calculated at the point $\left(\xi\left(t_{k}\right), \zeta\left(t_{k}\right)\right)$ while $P_{+}=$ $P\left(\xi\left(t_{k}^{+}\right), \zeta\left(t_{k}^{+}\right)\right), Q_{+}=Q\left(\xi\left(t_{k}^{+}\right), \zeta\left(t_{k}^{+}\right)\right)$

Lemma 2. Let $G(S, \rho): R \times R \rightarrow R$ be a one parameter family of $C^{2}$ map satisfying the following conditions:

$$
\text { (i) } G(0, \rho)=0, \quad(i i) \frac{\partial G}{\partial S}(0,0)=1, \quad(i i i) \frac{\partial^{2} G}{\partial S \partial \rho}(0,0)>0, \quad \text { (iv) } \frac{\partial^{2} G}{\partial S^{2}}(0,0)<0,
$$

then $G$ has two branches of fixed point near zero. The first branch is $S_{1}(\rho)=0$ for all $\rho$. The second bifurcating branch $S_{2}(\rho)$ changes its value from negative to positive as it increases through $\rho=0$ with $S_{2}(0)=0$. The fixed points of the first branch are stable if $\rho<0$ and unstable if $\rho>0$, while those of the bifurcating branch have the opposite stability.

\subsection{Properties of the ODE model}

Before investigating the dynamics of system (2) and their biological implications, we first need to have a clear view of the dynamics of the system without an impulsive control strategy which is given by model (1). Obviously, there are both a trivial equilibrium $E_{0}=(0,0)$ and a boundary equilibrium $E_{\eta}=(1 / \eta, 0)$ for system (2). Denote

$$
\left(x_{1}, y_{1}\right)=\left(\frac{c-q-\delta \omega+\sqrt{(c-q-\delta \omega)^{2}-4 q \omega \delta}}{2 q \omega}, \frac{r}{p}\left(1-\eta x_{1}\right)\right)
$$


and

$$
\left(x_{2}, y_{2}\right)=\left(\frac{c-q-\delta \omega-\sqrt{(c-q-\delta \omega)^{2}-4 q \omega \delta}}{2 q \omega}, \frac{r}{p}\left(1-\eta x_{2}\right)\right) .
$$
follows.

By simple analysis, the existence and stability of the equilibria of model (1) can be summarized as

Proposition 1. If $c-q-\delta \omega<2 \sqrt{q \omega \delta}$ or $c-q-\delta \omega \geq 2 \sqrt{q \omega \delta}$ and $x_{2} \geq 1 / \eta$ hold true, then for system (1) there just exist a trivial equilibrium $E_{0}=(0,0)$ and an equilibrium $E_{\eta}=(1 / \eta, 0)$ and the boundary equilibrium $E_{\eta}$ is globally stable. If $c-q-\delta \omega>2 \sqrt{q \omega \delta}$ and $x_{2}<1 / \eta \leq x_{1}$ hold true, apart from the equilibria $E_{0}$ and $E_{\eta}$, there would also appear an interior equilibrium $E_{2}=\left(x_{2}, y_{2}\right)$ which is globally stable, while the boundary equilibrium $E_{\eta}$ loses its stability. Especially, when $c-q-\delta \omega=2 \sqrt{q \omega \delta}$, then $x_{1}=x_{2}$. If $c-q-\delta \omega>2 \sqrt{q \omega \delta}$ and $1 / \eta>x_{1}$ hold true, then another positive equilibrium $E_{1}=\left(x_{1}, y_{1}\right)$ appears, which is a saddle point whenever it exists. The effector cell-free equilibrium $E_{\eta}$ and the positive equilibrium $E_{2}$ are bi-stable under these conditions.

Realistically, the population size of tumour cells will continuously grow and eventually reach its carrying capacity in the absence of combination treatment involving surgery and injection of effector cells. Therefore, we naturally assume that the interior equilibrium for model (1) does not exist, and in this paper we assume that $c-q-\delta \omega<2 \sqrt{q \omega \delta}$ holds true. For this case, there are two equilibria $E_{0}$ and $E_{\eta}$ for model (1) and the latter is globally stable. This indicates that the orbits of model (1) will approach the effector cell-free equilibrium $E_{\eta}$, as shown in Fig.1. The purpose of the comprehensive therapy is to control the tumour cells below a certain level which should obviously be below the carrying capacity. Thus it is reasonable to assume that $V_{L}<1 / \eta$.

In order to effectively inhibit tumour cell growth, comprehensive treatment strategies should be implemented once the density of tumour cells reach some given threshold level. Moreover, the proposed model (2) can help us to evaluate the effectiveness of combination therapy tactics, which we will address in more detail in the coming sections.

\section{Definition of the Poincaré map and its main properties}

In order to address the dynamical behaviour of system (2) and to discuss the integrated treatment strategies on the tumour cell control, we first investigate the complete dynamics of model (2).

Denote the vertical isocline of system (1) as

$$
L_{1}: y=\frac{r}{p}(1-\eta x), x \in[0,1 / \eta]
$$

and the vertical component of the intersection point $A$ of line $L_{1}$ with the line $x=(1-\theta) V_{L}$ is represented by $y_{h}$ with $y_{h}=\frac{r}{p}\left(1-\hat{\eta}(1-\theta) V_{L}\right)$, shown in Fig.1.

According to the definition of the impulsive function $I(x, y)$ we have

$$
I_{1}(x(t), y(t))=(1-\theta) x(t), \quad I_{2}(x(t), y(t))=\rho y(t)+\tau
$$

and

$$
I(x(t), y(t))=\left(I_{1}(x(t), y(t)), I_{2}(x(t), y(t))\right) .
$$

Let $\Phi\left(t, x_{0}, y_{0}\right)=\left(x\left(t, x_{0}, y_{0}\right), y\left(t, x_{0}, y_{0}\right)\right)$ be a solution of system (1) satisfying initial conditions $x\left(t_{0}, x_{0}, y_{0}\right)=x_{0}$ and $y\left(t_{0}, x_{0}, y_{0}\right)=y_{0}$. Define the two sections

$$
\Sigma_{\theta}=\left\{(x, y): x=(1-\theta) V_{L}, y \geq 0\right\}, \quad \Sigma_{V_{L}}=\left\{(x, y): x=V_{L}, y \geq 0\right\}
$$

and choose the section $\Sigma_{\theta}$ as the Poincaré section. For any initial value $\left((1-\theta) V_{L}, y_{0}\right) \in \Sigma_{\theta}$, there exists a unique solution of system (1), denoted by $\Phi_{T_{V_{L}}}\left(t,(1-\theta) V_{L}, y_{0}\right)$ with $T_{V_{L}}$ satisfying $\left.x\left(T_{V_{L}},(1-\theta) V_{L}\right), y_{0}\right)=$ $V_{L}$, intersecting with line $x=V_{L}$ at a point $\left(V_{L}, y_{V_{L}}\right)$. Therefore, we can define the function $\varphi$ as $\varphi\left(y_{0}\right)=y_{V_{L}}$ and then the Poincaré map $\phi$ should be the composite function of $\varphi$ and $I_{2}$, which is given as:

$$
\phi\left(y_{0}\right)=I_{2} \circ \varphi\left(y_{0}\right) .
$$


Theorem 1. The Poincaré map $\phi$ satisfies the following properties:

(I) $\phi$ is well defined in the interval $[0,+\infty)$ and the range of $\phi$ is $\left[\tau, \rho y_{h}+\tau\right]$. It is increasing on $\left[0, y_{h}\right)$ and decreasing on $\left[y_{h},+\infty\right)$;

(II) $\phi$ is continuously differentiable;

(III) $\phi$ is concave on $\left[0, y_{h}\right)$;

(IV) $\phi$ has a unique fixed point for $\tau>0$;

(V) $\phi$ is bounded with $\phi=\tau$ is the horizontal asymptote for $\phi$ as $y_{0} \rightarrow \infty$.

Proof. (I) It follows from Fig. 1 that any orbit initiating from the line $x=(1-\theta) V_{L}$ can finally approach the line $x=V_{L}$. Therefore the domain of $\phi$ is $[0,+\infty)$. For any $y_{01}, y_{02} \in\left[0, y_{h}\right)$ with $y_{01}<y_{02}$, according to the existence and uniqueness of solutions of system (1), the vertical components of both orbits $\Phi(t,(1-$ $\left.\theta) V_{L}, y_{01}\right)$ and $\Phi\left(t,(1-\theta) V_{L}, y_{02}\right)$ satisfy $y\left(t_{1},(1-\theta) V_{L}, y_{01}\right)<y\left(t_{2},(1-\theta) V_{L}, y_{02}\right)$, where $t_{i}(i=1,2)$ satisfying $x\left(t_{1},(1-\theta) V_{L}, y_{01}\right)=x\left(t_{2},(1-\theta) V_{L}, y_{02}\right)=V_{L}$. This means that $\phi\left(y_{01}\right)<\phi\left(y_{02}\right)$ holds true. If the initial values are in the interval $\left[y_{h},+\infty\right)$, i.e. $y_{01}, y_{02} \in\left[y_{h},+\infty\right)$ with $y_{01}<y_{02}$, then the orbits will first turn around the point $A$ and cross the line $x=(1-\theta) V_{L}$ at the points $\left((1-\theta) V_{L}, \tilde{y}_{01}\right)$ and $\left((1-\theta) V_{L}, \tilde{y}_{02}\right)$ on the segment $\left\{(x, y) \mid x=(1-\theta) V_{L}, 0<y<y_{h}\right\}$, respectively, and then hit the line $x=V_{L}$. Obviously, there must be $\tilde{y}_{01}>\tilde{y}_{02}$, then we have

$$
\phi\left(y_{01}\right)=\phi\left(\tilde{y}_{01}\right)>\phi\left(\tilde{y}_{02}\right)=\phi\left(y_{02}\right) .
$$

In conclusion, the Poincaré map $\phi$ increases in $\left[0, y_{h}\right)$ and decreases in $\left[y_{h},+\infty\right)$.

(II) To verify the continuous differentiability of $\phi$, we consider two cases, i.e. $y_{0} \in\left[0, y_{h}\right)$ and $y_{0} \in$ $\left[y_{h},+\infty\right)$. For $y_{0} \in\left[0, y_{h}\right)$, the property comes from the theorem of the differentiability of the solution of the ordinary differential equation with respect to its initial conditions. Denote

$$
P(x, y)=r x(t)(1-\eta x(t))-p x(t) y(t), \quad Q(x, y)=\frac{c x(t) y(t)}{1+\omega x(t)}-q x(t) y(t)-\delta y(t),
$$

and it is easy to see that if $y_{0} \in\left[0, y_{h}\right)$, then $P(x, y) \neq \theta$ for all $(x, y) \in\left\{(1-\theta) V_{L} \leq x \leq V_{L}, 0 \leq y<\right.$ $\left.y\left(t,(1-\theta) V_{L}, y_{h}\right)\right\} \doteq \Sigma_{(x, y)}$. Let

$$
h(x, y)=h\left(x, y\left(x, x_{0}, y_{0}\right)\right)=\frac{Q(x, y)}{P(x, y)}
$$

then we have

$$
\frac{\partial h}{\partial y}=\frac{\gamma x(1-\eta x)(c x-(q x+\delta)(1+\omega x))}{(1+\omega x)(r x(1-\eta x)-p x y)^{2}},
$$

which is continuous on $\Sigma_{(x, y)}$. Therefore $\frac{\partial y}{\partial y_{0}}=\exp \left(\int_{(1-\theta) V_{L}}^{V_{L}} \frac{\partial h}{\partial y} d x\right)$ is continuous on $y_{0} \in\left[0, y_{h}\right)$, which means that $\phi$ is continuously differential on $y_{0} \in\left[0, y_{h}\right)$. Moreover, the limitation

$$
\begin{aligned}
& \lim _{y_{0} \rightarrow y_{h}^{-}} \int_{(1-\theta) V_{L}}^{V_{L}}\left(\frac{1}{\left(r(1-\eta x)-p y_{0}\right)^{2}}\right) d x \\
= & \lim _{y_{0} \rightarrow y_{h}^{-}} \frac{1}{r \eta}\left(\frac{1}{r \eta(1-\theta)-r+p y_{0}}-\frac{1}{r \eta V_{L}-r+p y_{0}}\right)=+\infty,
\end{aligned}
$$

which indicates that $\lim _{y_{0} \rightarrow y_{h}^{-}} \exp \left(\int_{(1-\theta) V_{L}}^{V_{L}} \frac{\partial h}{\partial y} d x\right)=0$, i.e. the left derivative of $\phi$ at $y_{h}$ is equal to 0 .

For $y_{0} \in\left[y_{h},+\infty\right)$, any orbit starting from $\left((1-\theta) V_{L}, y_{0}\right)$ will first turn around the point $A$ and cross the line $x=(1-\theta) V_{L}$ on $y \in\left[0, y_{h}\right]$ at $\left((1-\theta) V_{L}, \tilde{y}_{0}\right)$, and then approach the line $x=V_{L}$. Define the function $\hbar: \hbar\left(y_{0}\right)=\tilde{y}_{0}$. Therefore, $\phi$ is the composite function of $\hbar$ and $\phi$ for $y \in\left[0, y_{h}\right)$. The continuous differentiability of $\phi$ for $y \in\left[0, y_{h}\right)$ is discussed above, and the function $\hbar$ is continuously differential because of the standard theory of Poincaré application (the Cauchy-Lipschitz theorem with parameter), which yields that $\phi$ is continuously differentiable for $y_{0} \in\left[y_{h},+\infty\right)$. And it is easy to verify that the right 
derivative of $\phi$ at $y_{h}$ is also equal to 0 . Therefore, $\phi$ is continuously differentiable at $y_{h}$ with $\left.\frac{d \phi}{d y_{0}}\right|_{y_{h}}=0$. Based on the above discussion, we can conclude that $\phi$ is continuously differentiable for $y_{0} \in[0,+\infty)$.

(III) For system (1), we can rewrite it in the following form in the phase space

$$
\left\{\begin{array}{l}
\frac{d y}{d x}=h(x, y) \\
y\left((1-\theta) V_{L}\right)=y_{0}
\end{array}\right.
$$

where $h(x, y)$ is given in equation (9), and equation (12) is equivalent to the following equation

$$
\left.y\left(x,(1-\theta) V_{L}, y_{0}\right)=y_{0}+\int_{(1-\theta) V_{L}}^{V_{L}} h\left(s, y\left(s,(1-\theta) V_{L}, y_{0}\right)\right)\right) d s .
$$

Thus, the Poincaré map for $y_{0} \in\left[0, y_{h}\right)$ can also be depicted as

$$
\phi\left(y_{0}\right)=\rho y\left(V_{L},(1-\theta) V_{L}, y_{0}\right)+\tau .
$$

Taking the second order derivative of $\phi$ with respect to $y_{0}$ yields

where

$$
\frac{d^{2} \phi}{d y_{0}^{2}}=\rho \frac{\partial^{2} y}{\partial y_{0}^{2}}=\rho \frac{\partial y}{\partial y_{0}} \int_{(1-\theta) V_{L}}^{V_{L}} \frac{\partial^{2} h}{\partial y^{2}} \frac{\partial y}{\partial y_{0}} d x
$$

$$
\frac{\partial y}{\partial y_{0}}=\exp \left(\int_{(1-\theta) V_{L}}^{V_{L}} \frac{\partial h}{\partial y} d x\right)^{0}
$$

and

$$
\left\{\begin{aligned}
\frac{\partial h}{\partial y} & =\frac{r x(1-\eta x)(c x-(q x+\delta)(1+\omega x))}{(1+\omega x)(r x(1-\eta x)-p x y)^{2}} \\
\frac{\partial^{2} h}{\partial y^{2}} & =\frac{2 r p x^{2}(1-\eta x)(c x-(q x+\delta)(1+\omega x))}{(r x(1-\eta x)-p x y)^{3}(1+\omega x)} .
\end{aligned}\right.
$$

Since $y<y_{h}$, we have $r x(1-\eta x)-p x y>0$ while $c x-(q x+\delta)(1+\omega x)<0$ for $c-q-\delta \omega<2 \sqrt{q \omega \delta}$. Thus, $\frac{\partial h}{\partial y}<0$ and $\frac{\partial^{2} h}{\partial y^{2}}<0$, which indicate that

$$
\frac{\partial^{2} y}{\partial y_{0}^{2}} \leq 0, y_{0} \in\left[0, y_{h}\right)
$$

Therefore, $y\left(V_{L},(1-\theta), y_{0}\right)$ is concave with respect to $y_{0}$ for $y_{0} \in\left[0, y_{h}\right)$, and consequently $\phi$ is concave in $\left[0, y_{h}\right)$.

(IV) As shown in (I), $\phi$ is decreasing on $\left[y_{h},+\infty\right)$, that means that $\phi\left(y_{0}\right)<\phi\left(y_{h}\right)=\rho y_{h}+\tau$ for $y_{0} \in$ $\left[y_{h},+\infty\right)$, which indicates that there exists a sufficiently large $\grave{y} \in\left[y_{h},+\infty\right)$ such that $\grave{y}>\rho y_{h}+\tau>\phi(\grave{y})$. Definitely, we have $\phi(0)=\tau>0$. It follows from the intermediate value theorem of continuous functions that there must be a $y^{*} \in[0, \tilde{y}]$ such that $\phi\left(y^{*}\right)=y^{*}$, that is, there exists a fixed point of $\phi$.

If $\phi\left(y_{h}\right)<y_{h}$, then the fixed point $y^{*}$ is on $\left[0, y_{h}\right)$. And the uniqueness of the fixed point on $\left[0, y_{h}\right)$ is guaranteed by the concavity of $\phi$. On the other hand, $\phi$ is decreasing on $\left[y_{h}, \infty\right)$ which leads to $\phi(y)<$ $\phi\left(y_{h}\right)<y_{h}$, then we have that there is no fixed point on $\left[y_{h},+\infty\right)$.

If $\phi\left(y_{h}\right) \geq y_{h}$, then the fixed point of $\phi$ does not lie in the interval $\left[0, y_{h}\right)$ according to the concavity of $\phi$ on $\left[0, y_{h}\right)$. This means that the fixed point is on the interval $\left[y_{h},+\infty\right)$. Furthermore, $\phi$ is decreasing on $\left[y_{h},+\infty\right)$. Therefore, there exists one and only one fixed point of $\phi$.

(V) First, we can claim that $\phi\left(\left[y_{h},+\infty\right)\right) \subset \phi\left(\left[0, y_{h}\right]\right)$ according to the topological structure of system (1). From the property $(I)$, we know $\phi$ is continuous on $\left[0, y_{h}\right]$ which guarantees $\phi$ is bounded for $y_{0} \in\left[0, y_{h}\right]$. As a conclusion, $\phi$ is bounded for $y_{0} \in[0,+\infty)$. Furthermore, $\phi$ is decreasing on $\left[y_{h},+\infty\right)$. Therefore, to verify that $\phi=\tau$ is the horizontal asymptote for $\phi$ as $y_{0} \rightarrow+\infty$, we just need to show that $\tau$ is the 
infimum of $\phi$ for $y_{0} \in\left[y_{h},+\infty\right)$. Otherwise, there exists a $\bar{y} \in\left(0, y_{h}\right)$ such that $\phi\left(y_{0}\right)>\phi(+\infty)=\phi(\bar{y})>\tau$ for $y_{0} \in\left[y_{h},+\infty\right)$. Then, it follows from the dynamics of the backwards system (i.e. $x_{1}(t)=x(-t), y_{1}(t)=$ $y(-t))$ that the orbit of the backwards system initiating from $B\left((1-\theta) V_{L}, \tilde{y}\right), \tilde{y} \in(0, \bar{y})$ will turn around the point $A$ and then approaches the line $x=(1-\theta) V_{L}$ at $C\left((1-\theta) V_{L}, y^{\prime}\right), y \in\left(y_{h},+\infty\right)$. This means that $\phi(\hat{y})=\phi(\tilde{y})<\phi(\bar{y})$, which is a contradiction. This completes the proof.

\section{Effector cell-free periodic solution and its bifurcation}

In this section, we consider the special case first, i.e. $\tau=0$. Letting $y(t)=0$ for all $t \in(0,+\infty)$ we have the following subsystem of system (2)

$$
\left\{\begin{array}{l}
\dot{x}=r x(t)(1-\eta x(t)), x<V_{L}, \\
x\left(t^{+}\right)=(1-\theta) x(t), \quad x=V_{L} .
\end{array}\right.
$$

Integrating the first equation of (19) with the initial condition $x\left(0^{+}\right)=(1-\theta) V_{L}$, yields

$$
x(t)=\frac{(1-\theta) V_{L}}{\eta(1-\theta) V_{L}+\left(1-\eta(1-\theta) V_{L}\right) e^{-r t}} .
$$

Letting $\frac{(1-\theta) V_{L}}{\eta(1-\theta) V_{L}+\left(1-\eta(1-\theta) V_{L}\right) e^{-r T}}=V_{L}$ and solving it with respect to $\mathrm{T}$ we get the period, denoted by $T_{V_{L}}^{0}$ with $T_{V_{L}}^{0}=-\frac{1}{r} \ln \frac{(1-\theta)\left(1-\eta V_{L}\right)}{1-\eta V_{L}+\eta \theta V_{L}}$. Therefore model (19) has a periodic solution, denoted by $\xi^{0}(t)$ and $\xi^{0}(t)=\frac{(1-\theta) V_{L}}{\eta(1-\theta) V_{L}+\left(1-\eta(1-\theta) V_{L}\right) e^{-r t}}$ with period $T_{V_{L}}^{0}$. This indicates that for system $(2)$ there exists a semitrivial periodic solution $\left(\xi^{0}(t), 0\right)$ (the so called effector cell-free periodic solution) and its stability is addressed below.

Since $a(x, y)=-\theta x, b(x, y)=(\rho-1) y, \Psi(x, y)=x-V_{L}$, a straightforward calculation yields

$$
\begin{gathered}
\frac{\partial P}{\partial x}=r-2 r \eta x-p y, \frac{\partial Q}{\partial y}=\frac{c x}{1+\omega x}-q x-\delta, \\
\frac{\partial a}{\partial x}=-\theta, \frac{\partial a}{\partial y}=0, \frac{\partial b}{\partial x}=0, \frac{\partial b}{\partial y}=\rho-1, \\
\frac{\partial \Psi}{\partial x}=1, \frac{\partial \Psi}{\partial y}=0
\end{gathered}
$$

and

$$
\triangle_{1}=\frac{P_{+}\left(\frac{\partial b}{\partial y} \frac{\partial \Psi}{\partial x}-\frac{\partial b}{\partial x} \frac{\partial \Psi}{\partial y}+\frac{\partial \Psi}{\partial x}\right)+Q+\left(\frac{\partial a}{\partial x} \frac{\partial \Psi}{\partial y}-\frac{\partial a}{\partial y} \frac{\partial \Psi}{\partial x}+\frac{\partial \Psi}{\partial y}\right)}{P \frac{\partial \Psi}{\partial x}+Q \frac{\partial \Psi}{\partial y}}=\frac{\rho(1-\theta)\left(1-\eta(1-\theta) V_{L}\right)}{1-\eta V_{L}} .
$$

Moreover, we have

$$
\begin{aligned}
\int_{0}^{T_{V_{L}}^{0}}\left(\frac{\partial P}{\partial x}+\frac{\partial Q}{\partial y}\right) d t= & \int_{0}^{T_{V_{L}}^{0}}\left(r-2 r \eta x_{T}(t)+\frac{c x_{T}(t)}{1+\omega x_{T}(t)}-q x_{T}(t)-\delta\right) d t \\
= & \left((r-\delta) t-\frac{q+2 r \eta}{r \eta} \ln \left(\eta(1-\theta) V_{L} e^{r t}+\left(1-\eta(1-\theta) V_{L}\right)\right)\right. \\
& \left.+\frac{c}{r(\omega+\eta)} \ln \left((\omega+\eta)(1-\theta) V_{L} e^{r t}+1-\eta(1-\theta) V_{L}\right)\right)\left.\right|_{0} ^{T_{V_{L}}^{0}} \\
= & -\ln \frac{(1-\theta)\left(1-\eta(1-\theta) V_{L}\right)}{1-\eta V_{L}}+\frac{\delta}{r} \ln \frac{(1-\theta)\left(1-\eta v_{L}\right)}{1-\eta(1-\theta) V_{L}}+ \\
& \frac{q}{r \eta} \ln \left(\frac{1-\eta V_{L}}{1-\eta(1-\theta) V_{L}}\right)+\frac{c}{r(\eta+\omega)} \ln \left(\frac{\left(1-\eta(1-\theta) V_{L}\right)\left(1+\omega V_{L}\right)}{\left(1-\eta V_{L}\right)\left(1+\omega(1-\theta) V_{L}\right)}\right)
\end{aligned}
$$

Denote

$$
A_{1}=\frac{\delta}{r} \ln \frac{(1-\theta)\left(1-\eta v_{L}\right)}{1-\eta(1-\theta) V_{L}}+\frac{q}{r \eta} \ln \left(\frac{1-\eta V_{L}}{1-\eta(1-\theta) V_{L}}\right)+\frac{c}{r(\eta+\omega)} \ln \left(\frac{\left(1-\eta(1-\theta) V_{L}\right)\left(1+\omega V_{L}\right)}{\left(1-\eta V_{L}\right)\left(1+\omega(1-\theta) V_{L}\right)}\right)
$$


then the Floquet multiplier $\mu_{2}$ can be calculated as

$$
\left|\mu_{2}\right|=\triangle_{1} \exp \left(\int_{0}^{T}\left(\frac{\partial P}{\partial x}+\frac{\partial Q}{\partial y}\right) d t\right)=\rho \exp \left(A_{1}\right)
$$

Thus, according to Lemma 1 we have the following conclusion.

Theorem 2. Let $\tau=0$, then there exists a semi-trivial order-1 periodic solution $\left(\xi^{0}(t), 0\right)$ of system (2) which is asymptotically stable if the following condition holds

$$
\rho \exp \left(A_{1}\right)<1
$$

Considering $A_{1}$ as a function of $\theta$ and taking the first order derivative of $A_{1}$ with respect to $\theta$, one has

$$
\begin{aligned}
A_{1}^{\prime}(\theta) & =-\frac{\delta}{r(1-\theta)(1-\eta)\left(1-\eta(1-\theta) V_{L}\right)}-\frac{q V_{L}}{r\left(1-\eta(1-\theta) V_{L}\right)}+\frac{c V_{L}}{r\left(1-\eta(1-\theta) V_{L}\right)\left(1+\omega(1-\theta) V_{L}\right)} \\
& =\frac{1}{r(1-\theta)\left(1-\eta(1-\theta) V_{L}\right)}\left(\frac{(1-\theta) V_{L}}{1+\omega(1-\theta) V_{L}}-q(1-\theta) V_{L}-\delta\right)
\end{aligned}
$$

It follows from $c-q-\delta \omega<2 \sqrt{q \omega \delta}$ and $V_{L}<1 / \eta$ that $A_{1}^{\prime}(\theta)<0$ holds true for all $\theta \in(0,1)$, which implies that $A_{1}(\theta)<A_{1}(0)=0$ for all $0<\theta<1$. Therefore, if we let $\rho=1$, then $\left|\mu_{2}\right|<1$ and further we have the following result.

Corollary 1. If $\tau=0$ and $\rho=1$, then the semi-trivial arder-1 periodic solution is globally asymptotically stable.

Proof. To show the global stability of the semi-trivial order-1 periodic solution $\left(\xi^{0}(t), 0\right)$, we only need to demonstrate its global attractivity. In fact, any solution initiating from $\left((1-\theta) V_{L}, y_{0}\right) \in \Sigma_{\theta}$ of model (2) with $y_{0} \leq y_{h}$ will meet this section infinite times; denote the vertical components of those points as $y_{k}(k=1,2, \cdots)$. It follows from $d y / d t<0$ in the domain $\{(x, y): 0<x<1 / \eta, y \geq 0\}$ that we have $y_{k+1}<y_{k}$ for all $k$. This means that $y_{k}$, will monotonically decrease and tend to 0. Moreover, if $y_{0}>y_{h}$ then it is easy to know that $y_{k}<y_{h}$ for all $k \geq 1$. These results confirm that the semi-trivial order-1 periodic solution is globally attractive. This completes the proof.

It is interesting to note that the stability of the semi-trivial order-1 periodic solution $\left(\xi^{0}(t), 0\right)$ will change at $\left|\mu_{2}\right|=1$. This shows that for model (2) there may exist a transcritical bifurcation at $\left|\mu_{2}\right|=1$. To illustrate this, we take $\left|\mu_{2}\right|$ as a function of $\rho$ with $\mu_{2}\left(\rho^{*}\right)=1$ and $\rho^{*}=\exp \left(-A_{1}\right)$. Letting $x_{0}=(1-\theta) V_{L}$, $y_{0}=S, S \in\left[0, y_{h}\right)$ and $\left(x, y\left(x, x_{0}, y_{0}\right)\right)$ be a solution of system $(1)$, then we have

$$
y\left(x, x_{0}, y_{0}\right)=y\left(x,(1-\theta) V_{L}, y_{0}\right)=y(x, S), x \in\left((1-\theta) V_{L}, V_{L}\right) .
$$

Taking the derivative of equation (27) with respect to $S$ on $y$ yields

$$
\frac{\partial y(x, S)}{\partial S}=\exp \left(\int_{(1-\theta) V_{L}}^{x} \frac{\partial}{\partial y}\left(\frac{Q(z, y(z, S))}{P(z, y(z, S))}\right) d z\right)
$$

and

$$
\frac{\partial^{2} y(x, S)}{\partial S^{2}}=\frac{\partial y(x, S)}{\partial S} \int_{(1-\theta) V_{L}}^{x} \frac{\partial^{2}}{\partial y^{2}}\left(\frac{Q(z, y(z, S))}{P(z, y(z, S))}\right) \frac{\partial y(x, S)}{\partial S} d z .
$$


If we consider the Poincaré map $\phi$ as a function of two parameters $S$ and $\rho$, then we have

$$
\begin{aligned}
\frac{\partial \phi(0, \rho)}{\partial S}= & \rho \frac{\partial y\left(V_{L}, 0\right)}{\partial S}=\exp \left(\int_{(1-\theta) V_{L}}^{x} \frac{\partial}{\partial y}\left(\frac{Q(z, y(z, S))}{P(z, y(z, S))}\right) d z\right) \\
= & \rho \exp \left(\int_{(1-\theta) V_{L}}^{V_{L}}\left(\frac{c z-(q z+\delta)(1+\omega z)}{r z(1-\eta z)(1+\omega z)}\right) d z\right) \\
= & \rho \exp \left(\left(\frac{c-q-\omega \delta}{r(\eta+\omega)} \ln \frac{1+\omega z}{1-\eta z}-\frac{\delta}{r} \ln z+\frac{\delta \eta}{r(\eta+\omega)} \ln (1-\eta z)+\right.\right. \\
& \left.\left.\frac{1+\omega}{r(\eta+\omega)} \ln (1+\omega z)+\frac{q}{r \eta} \ln (1-\eta z)+\frac{q}{r(\eta+\omega)} \ln \frac{1+\omega z}{1-\eta z}\right)\left.\right|_{(1-\theta) V_{L}} ^{V_{L}}\right) \\
= & \rho \exp \left(A_{1}\right) .
\end{aligned}
$$

Therefore, at the point $\left(0, \rho^{*}\right)$ we have

$$
\frac{\partial \phi\left(0, \rho^{*}\right)}{\partial S}=\rho^{*} \exp \left(A_{1}\right)=\left|\mu_{2}\left(\rho^{*}\right)\right|=1
$$

and

$$
\frac{\partial^{2} \phi\left(0, \rho^{*}\right)}{\partial S \partial \rho}=\exp \left(A_{1}\right)>0
$$

Further,

$$
\frac{\partial^{2} y(0, S)}{\partial S^{2}}=\frac{\partial y(0, S)}{\partial S} \int_{(1-\theta) V_{L}}^{V_{L}} g(z) \frac{\partial y(0, S)}{\partial S} d z
$$

where

$$
g(x)=\frac{2 p x(c x-(q x+\delta)(1+\omega x))}{(1+\omega x)(r x(1-\eta x))^{2}} .
$$

It is easy to see that $g(x)<0$ for all $x \in(0,4 \infty)$ due to $c-q-\delta \omega<2 \sqrt{q \omega \delta}$, which shows that $\frac{\partial^{2} \phi\left(0, \rho^{*}\right)}{\partial S^{2}}<0$. Obviously there is $\phi(0, \rho)=0$ for all $\rho \in(0,+\infty)$. Thus, it follows from Lemma 2 that we have the following conclusion.

Theorem 3. A transcritical bifurcation occurs at $\rho=\rho^{*}$ for the Poincaré map $\phi$. If $\rho<\rho^{*}$ then there exists a trivial fixed point, and when the parameter $\rho$ increases and exceeds $\rho^{*}$, then the Poincaré map possesses a positive fixed point. Consequently, for system (2) there exists an interior order-1 periodic solution when $\rho \in\left(\rho^{*}, \rho^{*}+\sigma\right)$ with $\sigma>0$.

In fact, from the Fig:2(a), we can deduce that when $\tau=0$ the curve of the Poincaré map would always intersect the line $y=y_{0}$ at $y_{0}=0$, that is, there is always a semi-trivial periodic solution which is stable when $\left.\frac{d \phi}{d y_{0}}\right|_{y_{0}=0}<1$, which is equivalent to $\left|\mu_{2}\right|<1$ according the equation (31), as shown in Fig.3(a). Moreover, if $\left|\mu_{2}\right|>1$, which means that $\left.\frac{d \phi}{d y_{0}}\right|_{y_{0}=0}>1$ through the equation (31), then a positive intersection point of the Poincaré map will appear on the line $y=y_{0}$. That means that there exists a positive order-1 periodic solution and the semi-trivial periodic solution will lose its stability (see Fig.3(b)). In conclusion, when $\tau=0$, there is a critical value $\rho^{*}$ such that when $\rho<\rho^{*}$, there just exists a semi-trivial periodic solution which is stable; when $\rho>\rho^{*}$, a positive order-1 periodic solution appears while the semi-trivial periodic solution loses its stability.

\section{Generalized results for the stability of the order- $k$ periodic solution}

\subsection{Generalized results}

The existence of the order-1 periodic solution of model (2) can be easily confirmed by Theorem 1, and also the stability of a positive order- 1 periodic solution for the special case (i.e. $\tau=0$ ) has been addressed in 
the last section. Thus, in this section we will focus on the stability of the positive order-1 periodic solution for $\tau>0$. Also, the complex dynamics are discussed.

Denote the order-1 periodic solution as $(\xi(t), \zeta(t))$. Without lose of generality, let $T_{P}$ be the period of $(\xi(t), \zeta(t))$, then we have

$$
\left(\xi\left(T_{P}\right), \zeta\left(T_{P}\right)\right)=\left(V_{L},\left(y^{*}-\tau\right) / \rho\right),\left(\xi\left(T_{P}^{+}\right), \zeta\left(T_{P}^{+}\right)\right)=\left((1-\theta) V_{L}, y^{*}\right) .
$$

Thus, there would be $\triangle_{1}=\frac{P_{+}\left((1-\theta) V_{L}, y^{*}\right)}{P\left(V_{L},\left(y^{*}-\tau\right) / \rho\right)}$ and

$$
\begin{aligned}
\int_{0}^{T_{P}}\left(\frac{\partial P}{\partial x}+\frac{\partial Q}{\partial y}\right) d t & =\int_{0}^{T_{P}}\left(r(1-2 \eta \xi(t))-p \zeta(t)+\frac{c \xi(t)}{1+\omega \xi(t)}-q \xi(t)-\delta\right) d t \\
& \doteq \int_{0}^{T_{P}} F(t) d t .
\end{aligned}
$$

Therefore, as a general result of the stability of the positive order-1 periodic solution, we have that if $\left|\triangle_{1}\right| \exp \left(\int_{0}^{T_{P}} F(t) d t\right)<1$ holds true, then the positive order-1 periodic solution $(\xi(t), \zeta(t))$ is locally stable according to Lemma 1.

To investigate the global stability of the positive order-k periodic solution, we define the infinite sequence $\left\{y_{n} \mid y_{n}=\phi^{n}\left(y_{0}\right)\right\}$ for all $y_{0} \in[0,+\infty)$. Then we will focus on the convergence of $\left\{y_{n}\right\}$, which indeed refers to the stability of the order- $k(k \geq 1)$ periodic solution of system (2).

Theorem 4. If $\phi\left(y_{h}\right)<y_{h}$, then the unique positive order-1 periodic solution of system (2) is globally asymptotically stable.

Proof. It follows from the property (IV) of the Poincaré map that there exists a unique fixed point of $\phi$ which is denoted as $y^{*}$. Consequently, system (2) has a unique positive order-1 periodic solution with initial condition $x_{0}=(1-\theta) V_{L}, y_{0}=y^{*}$. It follows from $\phi\left(y_{h}\right)<y_{h}$ and that $\phi$ is increasing on $\left[0, y_{h}\right)$ that $\phi\left(\left[0, y_{h}\right)\right) \subset\left[0, y_{h}\right)$. Therefore, the fixed point theorem guarantees $y^{*} \in\left[0, y_{h}\right)$.

For $y_{0} \in\left[0, y^{*}\right)$, it is easy to know that $\left\{y_{n}\right\}$ is monotonically increasing and bounded by $y^{*}$ due to the monotonicity and concavity of $\phi$. Therefore, $\lim _{\infty} y_{n}=y^{*}$ for $y_{0} \in\left[0, y^{*}\right)$. Similarly, we can conclude that $\left\{y_{n}\right\}$ is monotonically decreasing and converges to $y^{*}$ for $y_{0} \in\left(y^{*}, y_{h}\right]$.

For $y_{0} \in\left[y_{h},+\infty\right)$, we have $\phi\left(y_{0}\right)<\phi\left(y_{h}\right)<y_{h}$ since $\phi$ is decreasing on $\left[y_{h},+\infty\right)$. Thus, $\phi\left(\left[y_{h},+\infty\right)\right) \subset$ $\left[0, y_{h}\right)$. Then it follows from the case $y_{0} \in\left[0, y^{*}\right)$ that we have that $\left\{y_{n}\right\}$ will also converge to $y^{*}$ for $y_{0} \in\left[y_{h},+\infty\right)$. Therefore, we have $\lim _{n \rightarrow+\infty} y_{n}=y^{*}$ for all $y_{0} \in[0,+\infty)$, i.e. the order-1 periodic solution of model (2) is globally stable. The proof is completed.

Theorem 5. If $\phi\left(y_{h}\right)>y_{h}, \phi^{2}\left(y_{h}\right)>y_{h}$, then either the order-1 periodic solution is stable or there exists a stable order-2 periodic solution for system (2) which coexists with the order-1 periodic solution.

Proof. To verify this theorem, we only need to consider the limits of the sequence $\left\{y_{n}: y_{n}=\phi^{n}\left(y_{0}\right), y_{0} \in\right.$ $[0,+\infty)\}$. For $y_{0} \in\left[0, y_{h}\right], \phi$ is strictly monotonically increasing and $\phi$ has no fixed point on $\left[0, y_{h}\right]$, which implies that there must be an integer $m$ such that $\phi^{m-1}\left(y_{0}\right) \leq y_{h}$ and $\phi^{m}\left(y_{0}\right)>y_{h}$. Furthermore, $\phi$ is decreasing on $\left[y_{h}, \phi\left(y_{h}\right)\right]$ which yields the following condition

$$
\phi\left(\left[y_{h}, \phi\left(y_{h}\right)\right]\right)=\left[\phi^{2}\left(y_{h}\right), \phi\left(y_{h}\right)\right] \subset\left[y_{h}, \phi\left(y_{h}\right)\right],
$$

and there is also $\phi\left(\left(y_{h},+\infty\right)\right) \subset \phi([0,+\infty))$. All of these results guarantee that any sequence $\left\{y_{n}\right\}$ will finally enter into $\left[y_{h}, \phi\left(y_{h}\right)\right]$. In other words, there exists an integer $m$ such that $y_{n}=\phi^{n}\left(y_{0}\right) \in\left[y_{h}, \phi\left(y_{h}\right)\right], n \geq m$ for $y_{0} \in[0,+\infty)$. Therefore, we just need to consider the initial condition for $y_{0} \in\left[y_{h}, \phi\left(y_{h}\right)\right]$ and there are four different cases:

(i) $y_{1}>y_{0}>y_{2}$. In this case, we have $y_{3}=\phi\left(y_{2}\right)>\phi\left(y_{0}\right)=y_{1}$ and further $y_{4}=\phi\left(y_{3}\right)<\phi\left(y_{1}\right)=y_{2}$, so $y_{3}>y_{1}>y_{0}>y_{2}>y_{4}$. As a conclusion we obtain

$$
\cdots>y_{2 n+1}>y_{2 n-1}>\cdots>y_{3}>y_{1}>y_{0}>y_{2}>y_{4}>\cdots>y_{2 n}>y_{2 n+2}>\cdots .
$$

(ii) $y_{1}<y_{0}<y_{2}$. Performing the similar discussion to (i) yields

$$
\cdots<y_{2 n+1}<y_{2 n-1}<\cdots<y_{3}<y_{1}<y_{0}<y_{2}<y_{4}<\cdots<y_{2 n}<y_{2 n+2}<\cdots .
$$


(iii) $y_{1}>y_{2}>y_{0}$. In this scenario, we obtain that $\phi\left(y_{1}\right)=y_{2}<y_{3}=\phi\left(y_{2}\right)<\phi\left(y_{0}\right)=y_{1}$ and $\phi\left(y_{2}\right)=y_{3}>y_{4}=\phi\left(y_{3}\right)>\phi\left(y_{1}\right)=y_{2}$, which results in $y_{1}>y_{3}>y_{4}>y_{2}>y_{0}$. By induction, we derive

$$
y_{1}>y_{3}>\cdots>y_{2 n-1}>y_{2 n+1}>\cdots>y_{2 n+2}>y_{2 n}>\cdots>y_{4}>y_{2}>y_{0} .
$$

(iv) $y_{1}<y_{2}<y_{0}$. A similar process to (iii) yields

$$
y_{1}<y_{3}<\cdots<y_{2 n-1}<y_{2 n+1}<\cdots<y_{2 n+2}<y_{2 n}<\cdots<y_{4}<y_{2}<y_{0} .
$$

For both cases (i) and (ii), it is easy to see that there are two different limits $y_{1}^{*}$ and $y_{2}^{*}$ with $\left\{y_{2 n}\right\}$ converging to $y_{1}^{*}$ and $\left\{y_{2 n-1}\right\}$ tending to $y_{2}^{*}$. These conclusions imply that there is an order-2 periodic solution of system (2) which is stable. For cases (iii) and (iv), there is $y^{*} \in\left[y_{h}, \phi\left(y_{h}\right)\right]$ such that $\lim _{k \rightarrow+\infty} y_{2 k}=$ $\lim _{k \rightarrow+\infty} y_{2 k+1}=y^{*}$ or there exist $y_{1}^{*}$ and $y_{2}^{*}$ with $y_{1}^{*} \neq y_{2}^{*}$ such that $\lim _{k \rightarrow+\infty} y_{2 k}=y_{1}^{*}$ and $\lim _{k \rightarrow+\infty} y_{2 k-1}=y_{2}^{*}$, respectively. This completes the proof.

Theorem 5 gives a sufficient condition for the stability of the order- 1 or order- 2 periodic solution when $\phi\left(y_{h}\right)>y_{h}$. However, we still cannot confirm whether the order-1 periodic solution is globally stable or not under this condition. The next theorem is to determine a sufficient and necessary condition for the global stability of the order-1 periodic solution of model (2).

Theorem 6. When $\phi\left(y_{h}\right)>y_{h}$, then the order-1 periodic solution of system (2) is globally stable if and only if $\phi^{2}(y)>y$ for all $y \in\left[y_{h}, y^{*}\right)$ holds true.

Proof. When $\phi\left(y_{h}\right)>y_{h}$, it follows from Theorem 1 that there exists a fixed point $y^{*} \in\left[y_{h}, \phi\left(y_{h}\right)\right]$ such that $\phi\left(y^{*}\right)=y^{*}$. To verify the sufficiency, we consider three different cases: a) $y_{0} \in\left[y_{h}, y^{*}\right]$; b) $y_{0} \in\left[0, y_{h}\right)$; c) $y_{0} \in\left(y^{*},+\infty\right)$.

When $y_{0} \in\left[y_{h}, y^{*}\right]$, according to the property (I) of the Poincaré map $\phi$ there is $\phi\left(y_{0}\right)>\phi\left(y^{*}\right)=y^{*}$, which implies that there is $\phi^{2}\left(y_{0}\right)<\phi\left(y^{*}\right)=y^{*}$. Because of $\phi^{2}\left(y_{0}\right)>y_{0}$ we have $\phi^{2 k}\left(y_{0}\right), k=1,2 \ldots$ is strictly increasing and $\phi^{2 k}\left(y_{0}\right) \rightarrow y^{*}$ as $k \rightarrow+\infty$. Then according to the topological structure of system (1), the series $\phi^{2 k-1}\left(y_{0}\right)$ is decreasing and tends to $y^{*}$ as $k \rightarrow+\infty$. Therefore, we have that $\phi^{n}\left(y_{0}\right) \rightarrow y^{*}$ as $n \rightarrow+\infty$.

If $y_{0} \in\left[0, y_{h}\right)$, then there is $\phi\left(y_{0}\right)>y_{0}$ through the properties of the Poincaré map. Moreover, $\phi$ is increasing for $y_{0} \in\left[0, y_{h}\right)$ which yields that there must be an integral $m$ such that $\phi^{m}\left(y_{0}\right) \in\left[y_{h}, y^{*}\right]$, then it follows from the former case that $\phi^{n}$ tends to $y^{*}$, or $\phi^{m}\left(y_{0}\right) \in\left[y^{*}, \phi\left(y_{h}\right)\right]$ which implies that there is $\bar{y} \in\left[y_{h}, y^{*}\right]$ such that $\phi^{m}\left(y_{0}\right)=\phi(\bar{y})$, then it follows for case a) that we have that $\phi^{n}\left(y_{0}\right) \rightarrow y^{*}$ as $n \rightarrow \infty$. Therefore, there would always be $\lim _{n \rightarrow+\infty} \phi^{n}\left(y_{0}\right)=y^{*}$ for all $y_{0} \in\left[0, y_{h}\right)$.

For case c), it follows from the topological structure of system (1) that any orbit initiating from $\left((1-\theta) V_{L}, y_{0}\right)$ will first turn around the point $A$ and hit the line $x=(1-\theta) V_{L}$ in the segment $\{(x, y) \mid x=$ $\left.(1-\theta) V_{L}, 0<y<y_{h}\right\}$. Therefore, there must be a $\tilde{y} \in\left[0, y_{h}\right)$ such that $\phi\left(y_{0}\right)=\phi(\tilde{y})$, then according to case b) we have that $\phi^{n}\left(y_{0}\right) \rightarrow y^{*}$ as $n \rightarrow+\infty$ for all $y_{0} \in\left(y^{*},+\infty\right)$.

Next, we consider if the positive order-1 periodic solution is globally stable, then there is $\phi^{2}(y)>y$ for all $y_{0} \in\left[y_{h}, y^{*}\right)$. Otherwise, there exists a $\check{y} \in\left[y_{h}, y^{*}\right)$ such that $\phi^{2}(\check{y}) \leq \check{y}$. When $\phi^{2}(\check{y})=\check{y}$ holds true, then $\check{y}$ is a fixed point of $\phi^{2}$ which is a positive order-2 periodic solution of system $(2)$. When $\phi^{2}(\check{y})<\check{y}$, there must be a $\hat{y} \in[0, \check{y}]$ such that $\phi^{2}(\hat{y})=\hat{y}$ since $\phi^{2}(0)>0$ and $\phi^{2}$ is continuous in $[0,+\infty)$, that means that there is a positive order- 2 periodic solution of system (2). And these are contradictory to the global stability of the positive order-1 periodic solution. This completes the proof.

In the following we give other sufficient conditions for the stability of the order-1 periodic solution of model (2) when $\rho=1$.

Theorem 7. There exists a pair of threshold values $p_{c}$ and $\omega_{c}$ such that the unique order-1 periodic solution of model (2) is globally stable for $p<p_{c}, \omega>\omega_{c}$.

Proof. Because of the assumption $c-q-\delta \omega<2 \sqrt{q \delta \omega}, Q(x, y)<0$ holds for $x<1 / \eta$. Moreover, according to the assumption $V_{L}<1 / \eta$, we have that $P(x, y)>0$ always hold true in the region $\left\{(x, y) \mid(1-\theta) V_{L}<\right.$ 
$\left.x<V_{L}\right\}$. Therefore, we have

$$
\frac{Q(x, y)}{P(x, y)}<\frac{Q(x, y)}{r x(1-\eta x)}
$$

Considering the following model

$$
\left\{\begin{array}{l}
\frac{d Y}{d x}=\frac{\frac{c x Y}{1+\omega x}-q x Y-\delta Y}{r x(1-\eta x)}, \\
Y\left((1-\theta) V_{L}\right)=y_{h},
\end{array}\right.
$$

and solving it for $x \in\left[(1-\theta) V_{L}, V_{L}\right]$, we have

$$
\begin{aligned}
Y(x)= & y_{h}\left(\frac{(1-\theta) V_{L}}{x}\right)^{\frac{\delta}{r}}\left(\frac{1-\eta x}{1-\eta(1-\theta) V_{L}}\right)^{\frac{q \omega+\delta \eta^{2}}{r \eta(\eta+\omega)}}\left(\frac{1+\omega x}{1+\omega(1-\theta) V_{L}}\right)^{\frac{q+\delta \omega}{r(\omega+\eta)}} \\
& \left(\frac{(1+\omega x)\left(1-\eta(1-\theta) V_{L}\right)}{(1-\eta x)\left(1+\omega(1-\theta) V_{L}\right)}\right)^{\frac{c-q-\omega \delta}{r(\eta+\omega)}} .
\end{aligned}
$$

Substituting $x=V_{L}$ into equation (41) yields

$$
\begin{aligned}
Y\left(V_{L}\right)= & y_{h}(1-\theta)^{\frac{\delta}{r}}\left(\frac{1-\eta V_{L}}{1-\eta(1-\theta) V_{L}}\right)^{\frac{q \omega+\delta \eta^{2}}{r \eta(\eta+\omega)}}\left(\frac{1+\omega V_{L}}{1+\omega(1-\theta) V_{L}}\right)^{\frac{q+\delta \omega}{\eta(\omega+\eta)}} \\
& \left(\frac{\left(1+\omega V_{L}\right)\left(1-\eta(1-\theta) V_{L}\right)}{\left(1-\eta V_{L}\right)\left(1+\omega(1-\theta) V_{L}\right)}\right)^{\frac{c-q-\omega \delta}{r(\eta+\omega)}} \\
\doteq & y_{h} M .
\end{aligned}
$$

It is easy to see that

$$
\lim _{\omega \rightarrow+\infty} M=(1-\theta)^{\frac{\delta}{r}}\left(\frac{1-\eta V_{L}}{1-(1-\theta) \eta V_{L}}\right)^{\frac{q+\eta \delta}{r \eta}}<1 .
$$

Therefore, there exists a sufficiently large $\omega_{c}$ such that $M<1$ for $\omega>\omega_{c}$. Then, we can conclude that if $\omega>\omega_{c}$

$$
\begin{aligned}
\lim _{p \rightarrow 0^{+}}\left(y_{h}-Y\left(V_{L}\right)-\tau\right) & =\lim _{p \rightarrow 0^{+}}\left(y_{h}(1-M)-\tau\right) \\
& =\lim _{p \rightarrow 0^{+}}\left(\frac{r}{p}\left(1-\eta(1-\theta) V_{L}\right)(1-M)-\tau\right)=+\infty
\end{aligned}
$$

This means that there is a threshold $p_{c}$ such that $y_{h}-Y\left(V_{L}\right)>0$ for $p<p_{c}$ when $\omega>\omega_{c}$. Furthermore, it follows from the comparison theorem of scaler differential equations and the theorem of Cauchy and Lipschitz with parameters that for $p<p_{c}$ and $\omega>\omega_{c}$, we have

$$
y_{h}-y_{V_{L}}\left(y_{h}\right)>y_{h}-Y\left(V_{L}\right)>\tau,
$$

where $y_{V_{L}}\left(y_{h}\right)$ is the vertical component of the intersection point of line $x=V_{L}$ to the orbit of system (1) initiating from the point $\left((1-\theta) V_{L}, y_{h}\right)$. Therefore, according to Theorem 4 the unique positive periodic solution of system (2) is globally stable. This completes the proof.

\subsection{Special case with the first integral}

In the last subsection, the sufficient or sufficient and necessary conditions for the stability of positive order1 or order-2 periodic solutions have been discussed through the Poincaré map. Those results confirm that the definition of the Poincaré map and its properties play important roles in the analysis of the model with state dependent feedback control. In particular the analytical formula of the Poincaré map is useful for evaluating those conditions provided in previous sections. Next, we will focus on the special case, i.e. $\eta=0$ which shows that the tumour cells will grow exponentially without control measures. 
Letting $\eta=0$ and taking any two points $(x, y)$ and $\left(x_{c}, y_{c}\right)$ in the same orbit of model (1), one has the following first integral of system (1)

$$
\int_{x_{c}}^{x}\left(\frac{c}{1+\omega z}-\frac{\delta}{z}-q\right) d z=\int_{y_{c}}^{y}\left(\frac{r}{z}-p\right) d z
$$

re-arranging it yields

$$
H(x, y)=-\frac{c}{\omega} \ln (1+\omega x)+\delta \ln (x)+q x+r \ln (y)-p y=h,
$$

where $h=H\left(x_{c}, y_{c}\right)$ is a constant.

According to the definition of the Lambert $\mathrm{W}$ function (Appendix A) and solving $H(x, y)=h$ with respect to $y$, we have

$$
y_{l}=-\frac{r}{p} W\left[0,-\frac{p}{r} \exp \left(\frac{c \ln (1+\omega x)-\delta \omega \ln (x)-q \omega x+h \omega}{r \omega}\right)\right]
$$

and

$$
y_{u}=-\frac{r}{p} W\left[-1,-\frac{p}{r} \exp \left(\frac{c \ln (1+\omega x)-\delta \omega \ln (x)-q \omega x+h \omega}{r \omega}\right)\right]
$$

From the properties of the Lambert $\mathrm{W}$ function, we have that $y_{l}$ and $y_{u}$ are well defined provided that

$$
-\frac{p}{r} \exp \left(\frac{c \ln (1+\omega x)-\delta \omega \ln (x)-q \omega x+h \omega}{r \omega}\right) \geq-e^{-1} .
$$

Lemma 3. When $\eta=0$, then the Poincaré map of model (2) can be well defined through the Lambert $W$ function, which is given as

$$
\phi\left(y_{0}\right)=-\frac{\rho r}{p} W\left[0,-\frac{p}{r} \exp \left(\frac{c \ln \left(1+\omega V_{L}\right)-\delta \omega \ln \left(V_{L}\right)-q \omega V_{L}+h_{0} \omega}{r \omega}\right)\right]+\tau
$$

with $h_{0}=H\left((1-\theta) V_{L}, y_{0}\right)$.

Proof. Considering the orbit initiating from the point $\left(x_{c}, y_{c}\right)=\left((1-\theta) V_{L}, y_{0}\right)$, which will reach a point $(x, y)=\left(V_{L}, y_{V_{L}}\right) \in \Sigma_{V_{L}}$, then the inequality (48) is equivalent to the following inequality

$$
\ln \frac{p}{r}+\frac{c}{r \omega} \ln \frac{1+\omega V_{L}}{1+\omega(1-\theta) V_{L}}+\frac{\delta}{r} \ln (1-\theta)-\frac{q \theta V_{L}}{r}+\ln y_{0}-\frac{p}{r} y_{0} \leq-1 .
$$

Define the function

$$
f(\theta)=\ln \frac{p}{r}+\frac{c}{r \omega} \ln \frac{1+\omega V_{L}}{1+\omega(1-\theta) V_{L}}+\frac{\delta}{r} \ln (1-\theta)-\frac{q \theta V_{L}}{r}+\ln y_{0}-\frac{p}{r} y_{0},
$$

from which we have

$$
f^{\prime}(\theta)=\frac{1}{r(1-\theta)}\left(\frac{c(1-\theta) V_{L}}{1+\omega(1-\theta) V_{L}}-q(1-\theta) V_{L}-\delta\right) .
$$

It follows from $c-q-\delta \omega<2 \sqrt{q \omega \delta}$ and $f^{\prime}(\theta)<0$ for any $\theta \in[0,1)$ that

$$
f(\theta)<f(0)=\ln \frac{p}{r}+\ln y_{0}-\frac{p}{r} \leq \ln \frac{p}{r}+\ln \frac{r}{p}-\frac{p}{r} \times \frac{r}{p}=-1 .
$$

This confirms that for any $x_{c}=(1-\theta) V_{L}, y_{c}=y_{0} \in[0,+\infty)$ and $x=V_{L}$ the inequality (48) always holds true. Therefore, $y_{V_{L}}$ can be well defined by the Lambert $\mathrm{W}$ function. Moreover, according to the property $(\mathrm{V})$ of the Poincaré map $\phi$, we know that $\phi$ is bounded for $y_{0} \in[0,+\infty)$, which indicates that $y_{V_{L}}$ should be defined by the branch $W[0, z]$, i.e.

$$
y_{V_{L}}=\varphi\left(y_{0}\right)=-\frac{r}{p} W\left[0,-\frac{p}{r} \exp \left(\frac{c \ln \left(1+\omega V_{L}\right)-\delta \omega \ln \left(V_{L}\right)-q \omega V_{L}+h_{0} \omega}{r \omega}\right)\right]
$$


with $h_{0}=H\left((1-\theta) V_{L}, y_{0}\right)$. Consequently, the Poincaré map would be

$$
\phi\left(y_{0}\right)=-\frac{\rho r}{p} W\left[0,-\frac{p}{r} \exp \left(\frac{c \ln \left(1+\omega V_{L}\right)-\delta \omega \ln \left(V_{L}\right)-q \omega V_{L}+h_{0} \omega}{r \omega}\right)\right]+\tau .
$$

This completes the proof.

Note that $y_{h}=\frac{r}{p}$ when $\eta=0$. Thus, $\phi\left(y_{h}\right)$ can be easily calculated as

$$
\phi\left(y_{h}\right)=-\frac{\rho r}{p} W\left[0,-\frac{p}{r} \exp \left(\frac{c \ln \left(1+\omega V_{L}\right)-\delta \omega \ln \left(V_{L}\right)-q \omega V_{L}+h_{1} \omega}{r \omega}\right)\right]+\tau
$$

where $h_{1}=H\left((1-\theta) V_{L}, \frac{r}{p}\right)$.

Similarly, we have

$$
\phi^{2}\left(y_{0}\right)=-\frac{\rho r}{p} W\left[0,-\frac{p}{r} \exp \left(\frac{c \ln \left(1+\omega V_{L}\right)-\delta \omega \ln \left(V_{L}\right)-q \omega V_{L}+h_{01} \omega}{r \omega}\right)\right]+\tau
$$

and

$$
\phi^{2}\left(y_{h}\right)=-\frac{\rho r}{p} W\left[0,-\frac{p}{r} \exp \left(\frac{c \ln \left(1+\omega V_{L}\right)-\delta \omega \ln \left(V_{L}\right)-q \omega V_{L}+h_{11} \omega}{r \omega}\right)\right]+\tau
$$

with $h_{01}=H\left((1-\theta) V_{L}, \phi\left(y_{0}\right)\right)$ and $h_{11}=H\left((1-\theta) V_{L}, \phi\left(y_{h}\right)\right)$, respectively

Letting $\phi\left(y_{h}\right)<y_{h}$ yields the following inequality:

$$
\tau-\frac{\rho r}{p} W\left[0,-\frac{p}{r} \exp \left(\frac{c \ln \left(1+\omega V_{L}\right)-\delta \omega \ln \left(V_{L}\right)-q \omega V_{L}+h_{1} \omega}{r \omega}\right)\right]<\frac{r}{p}
$$

which means that

$$
W\left[0,-\frac{p}{r} \exp \left(\frac{c \ln \left(1+\omega V_{L}\right)-\delta \omega \ln \left(V_{L}\right)-q \omega V_{L}+h_{1} \omega}{r \omega}\right)\right]>\frac{p \tau-r}{\rho r} .
$$

It follows from the properties of the Lambert $\mathrm{W}$ function that the inequality (59) is equivalent to

$$
\frac{p \tau-r}{r \rho} \exp \left(\frac{p \tau-r}{r \rho}\right)<-\frac{p}{r} \exp \left(\frac{c \ln \left(1+\omega V_{L}\right)-\delta \omega \ln \left(V_{L}\right)-q \omega V_{L}+h_{1} \omega}{r \omega}\right),
$$

substituting $h_{1}$ into inequality (60) and re-arranging it we have

$$
\frac{r-p \tau}{p \rho} \exp \left(\frac{p \tau-r}{r \rho}\right)>\exp \left(\frac{c \ln \left(\frac{1+\omega V_{L}}{1+\omega(1-\theta) V_{L}}\right)+\delta \omega \ln (1-\theta)-q \omega \theta V_{L}+r \ln \frac{r}{p}-r}{r \omega}\right) .
$$

Naturally, $\phi\left(y_{h}\right)>y_{h}$ is equivalent to

$$
\frac{r-p \tau}{p \rho} \exp \left(\frac{p \tau-r}{r \rho}\right)<\exp \left(\frac{c \ln \left(\frac{1+\omega V_{L}}{1+\omega(1-\theta) V_{L}}\right)+\delta \omega \ln (1-\theta)-q \omega \theta V_{L}+r \ln \frac{r}{p}-r}{r \omega}\right) .
$$

Also, we can calculate that $\phi^{2}\left(y_{h}\right)>y_{h}$ decided the following inequality

$$
\frac{r-p \tau}{p \rho} \exp \left(\frac{p \tau-r}{r \rho}\right)<\exp \left(\frac{c \ln \left(\frac{1+\omega V_{L}}{1+\omega(1-\theta) V_{L}}\right)+\delta \omega \ln (1-\theta)-q \omega \theta V_{L}+r \ln \phi\left(y_{h}\right)-p \phi\left(y_{h}\right)}{r \omega}\right) .
$$

Based on the above discussion, we have the following corollary.

Corollary 2. When $\eta=0$, if the inequality (61) holds true, then there exists a unique order-1 periodic solution of system (2) which is globally stable. If the inequalities (62) and (63) hold true, then either the order-1 periodic solution is stable or there exists a stable order-2 periodic solution for system (2) which coexists with the order-1 periodic solution.

Remark. It follows from the inequality (59) that there exists a critical value of $\rho$, denoted by $\rho_{c}$ with

$$
\rho_{c}=\frac{p \tau-r}{r W\left[0,-\frac{p}{r} \exp \left(\frac{\left.c \ln \left(1+\omega V_{L}\right)-\delta \omega \ln \left(V_{L}\right)-q \omega V_{L}+h_{1} \omega\right)}{r \omega}\right)\right]},
$$


such that $\phi\left(y_{h}\right)<y_{h}$ for all $\rho<\rho_{c}$. Therefore, the positive order-1 periodic solution is globally stable if $\rho<\rho_{c}$ when $\eta=0$.

\subsection{Complexity}

From the above subsection, we know that there exists a positive order- 1 or order- 2 periodic solution based on different conditions. To verify these conclusions numerically, we first considered the properties of $\phi\left(y_{0}\right)$, $\phi^{2}\left(y_{0}\right), \phi\left(y_{h}\right)$ and $\phi^{2}\left(y_{h}\right)$ by considering them as the functions of parameter $\rho$ and fixed all the other parameter values as: $\eta=0, r=5, p=0.8, c=0.3, q=0.23, \omega=1, \delta=0.3, \theta=0.2, \tau=1, V_{L}=5$. The relationship of $\phi\left(y_{h}\right)$ and $\phi^{2}\left(y_{h}\right)$ to parameter $\rho$ is shown in Fig.2(b). From Fig.2(b), we can see that $\phi\left(y_{h}\right)<y_{h}$ holds true when $0<\rho<1.2$, then according to Corollary 2 the positive order-1 periodic solution is globally stable which is shown in Fig.4(a). Simultaneously, we find that when $1.2<\rho<3.1$, then there are $\phi\left(y_{h}\right)>y_{h}$ and $\phi^{2}\left(y_{h}\right)>y_{h}$. Therefore, according to Corollary 2 there are stable positive order-1 ( shown in Fig.4(b)) or order-2 (shown in Fig.4(c)) periodic solutions with $\rho$ is chosen as 2 and 3 , respectively. In detail, Fig.4(b) shows us that $y_{0}<y_{2}<y_{1}$ holds true and then the positive order-1 periodic solution is stable while Fig.4(c) shows that when $y_{2}<y_{0}<y_{1}$ holds true, then there exists a positive order-2 periodic solution which is stable. However, if we choose $\rho=4$, then the attractor presents a complex situation as shown in Fig.4(d), which implies that higher order periodic solutions of system (2) may exist.

To illustrate the complexity, we chose $\eta=0.01$ and $\rho$ as the bifurcation parameter and the change of attractor of system (2) is shown in Fig.5. From Fig.5, we can see that when $\rho$ is relatively small, then there exists an order-1 periodic solution which is stable as shown in Fig.6(a). As $\rho$ increases, a stable order-2 periodic solution appears (see Fig.6(b)). When $\rho$ continuously increases, a stable order- 4 periodic solution appears which can be also seen in Fig.6(c). Simultaneously, it is easy to see from Fig.5 that the order-8 periodic solution can exist which is also shown in Fig.6(d). It is interesting to note that periodic solutions are associated with the regular variation of tumour population size in clinic. Based on the period of the periodic solution (denoted by $T$ ) the comprehensive therapy could be carried out every $T$ time without surveying the tumour population size. Furthermore, when $\rho$ increases to a certain level, then chaos appears. This chaotic behaviour is related to the irregular and unpredictable variation of tumour population size in clinic, which brings difficulty to survey the tumour population size and hence brings trouble to initiate the comprehensive therapy.

\section{Discussion and biological implications}

In this paper, we proposed a mathematical model to investigate how comprehensive therapy including surgery and immunotherapy affects the tumour system. By taking state-dependent feedback therapy strategies into consideration, the proposed model was formulated to be an impulsive model of the tumour immune system to describe how comprehensive therapy strategies are only implemented when the tumour cell population size reaches a critical level. Our density-dependent impulsive differential equations provide a more realistic description than existing continuous models [Arcietro et al., 2004; Kuznetsov \& Knott, 2001; Kuznetsov et al., 1994; Wilson \& Levy, 2012].

Theoretically analyzing this model, we find that it results in rich dynamics which also provide natural descriptions of real life problems. First of all, the dynamics of the ordinary differential equation model of the immune tumour system without any therapy was discussed and, in conclusion, it can present different cases: (a) there is no positive equilibrium; (b) there is only one positive equilibrium; (c) there are two positive equilibria, of which one is locally stable and the other is an unstable saddle point. For this paper, we just focused on case (a) when there is no interior equilibrium, a condition allowing the tumour cells to steadily increase to their carrying capacity if no therapy is carried out. Under this scenario, the Poincaré map possesses several important properties including the continuous differentiability on the phase set, its monotonicity, its concavity and the existence of the fixed point. It is easy to see from the properties of the Poincaré map that when $\tau=0$, a semi-trivial order-1 periodic solution always exists and a positive order-1 periodic solution can appear when some conditions hold. Further, we investigated the existence of the positive order- 1 and order- 2 periodic solutions when $\tau>0$ by using the properties of the Poincaré 
map. Also, we have numerically proved the existence of the positive order- 4 and order- 8 periodic solutions and chaos. It is interesting to see that the first integral of the ODE model exists if we assume $\eta=0$, and then all the conditions of Theorems 5 and 6 can be verified with anaclitic formulae by using the definition of the Lambert $\mathrm{W}$ function.

It is important to emphasize that the abundant dynamic phenomena suggest many interesting biological meanings, which can explain the experimental and clinical results and further show the efficiency of the therapy strategies for patients. In particular, if we carry out the therapy of surgery only, i.e. $\tau=0$ and $\rho=1$, then a semi-trivial periodic solution, which is globally stable, always exists. This result is in good agreement with the conclusions of other by researchers [Duffey et al., 2004; Herring et al., 2001; Walther et al., 1999] that if only surgery was performed the tumour cells could be controlled at a chosen level and no metastases would occur in the patients. It indicates that tumour cells persist while there is no immune memory. Obviously, this is not the ideal result. Fortunately, when additional therapy (i.e. immunotherapy) is carried out, it follows from Theorems 3 and 4 that the positive periodic solution can be feasible, meaning that clinically, the tumour cells can be controlled below a chosen level as well as the immune system retaining its activity. Therefore, we can conclude that comprehensive therapy with impulsive strategies is an effective way to both control cancers and keep the immune system active. Moreover, according to period of the periodic solution the density-dependent impulsive therapy regime could be converted into fixed-time pulsed therapy. Then with appropriate parameter values, guaranteeing existence of periodic solution, the treatment is actually pulsed treatment with frequency of inverse of period.

The existence of a globally stable positive order-1 periodic solution means that periodic therapy could be possible. During the progress of periodic therapy, key factors determining its success or failure are the therapy intensity per therapy application and how often the treatment is needed. To investigate how the therapy period will change with different therapy strategies, we let the three therapy parameters (including the instantaneous rate of resection for tumour cells $\theta$, the injection rate of effector cells $\tau$ and the growth rate of the effector cells $\rho$ ) change and fixed all other parameter yalues as: $r=5, \eta=0.01, p=0.8, c=0.3, \omega=$ $1, q=0.23, \delta=0.3$. The relationship between the treatment frequency and the treatment specifications is shown in Fig.7. It follows from Fig.7 that treatment frequency becomes low as one or other of the parameters $\tau, \theta$ and $\rho$ increases. Therefore, if we enhance the treatment strength each time, the necessary treatment frequency will become low, which may decrease the harm done to the patients. However, from Fig. 5 we can see that chaos may exist when $\rho$ is relatively bigger, which means that there is no regularity regarding the increasing of the tumour cells. This implies that it would be very difficult to survey the tumour in time and so the tumour cells could have exceeded the chosen level before the results of imaging studies, thus successful comprehensive therapy could not be implemented in time. Therefore, there might be an optimal value of treatment intensity such that, on the one hand, the treatment frequency is as low as possible, and on the other hand, the tumour cells can be properly surveyed.

In this paper, we mainly focused on a case where no interior equilibrium is feasible for system (1). However, for the case where two possible positive equilibria are possible, the dynamic behaviours would present much more varied phenomena, including the domain and the range of the Poincaré map being very complex. In partieular, the discussion of the existence and stability of the positive order- 1 or order- 2 periodic solutions could be divided into many different situations dependent on the values of $V_{L}$ and $\theta$. Also, there may exist a positive order-3 periodic solution which will lead to any order periodic solution occurring in such scenario. Moreover, it is worth mentioning that under such a situation the tumour cells can be controlled at a certain level after a finite number of comprehensive therapeutic interventions. All of these situations can guide different therapy strategies for controlling cancers, as shown in Fig.8. In Fig.8(a), a positive order- 2 periodic solution exists which implies that infinite numbers of therapeutic interventions should be carried out to control the cancer. However, if we change the control parameters (including the threshold level $V_{L}$ and the growth rate of the effector cells $\rho$ ), then the trajectory will finally tend to the steady state after finite control applications as shown in Fig.8(b) and Fig.8(c). This is associated with fact that after finite times of comprehensive therapy, tumour cells and effector cells will approach to a steady state with the tumour population size below the critical value. Moreover, by choosing the same parameter values but different initial densities of the tumour cells and effector cells from those in Fig.8(c), Fig.8(d) shows that no therapy is needed to control the tumour below a certain level. These differences mean that 
some patients may not need any therapy to maintain their tumour size below a certain level while others would need several repetitions of treatment to control the cancer, which are in good agreement with the clinical results of [Duffey et al., 2004; Herring et al., 2001; Walther et al., 1999]. Therefore, an individualized therapy should be recommended, which means that the optimal choice of treatment strategy for a given patient would depend on the density of the tumour cells and the effector cell population size at the outset.

It is worthy noticing that this study is of theoretical investigation on cancer dynamics under comprehensive therapy. By applying the threshold of the tumour population size $V_{L}$ we can formulate the impulsive differential equations to represent the density-dependent treatment. There a gap between theoretical model and the fact of the tumour size dependent-surgery, but we hope the approaches we used are able to induce more precise cancer therapy, and of course more reasonable mathematical models will be developed in the future. We obtained various cancer dynamics by choosing control parameters (including the threshold level $V_{L}$ and the growth rate of the effector cells $\rho$ ). It is ideal to estimate the unknown parameters based on the cohort data on tumour cells and effector cells, and by using this proposed methodology we may predicate the trend of development of tumour population size and assess efficacy of the therapy regime. However, the method of parameter estimation for the density-dependent impulsive differential equations rêmains unclear. Therefore, determining these control parameters by using our model is challenging and we leave this for future work.

Acknowledgements This work was supported by the National Natural Science Foundation of China (NSFC 11171268 (YX), $11571273(\mathrm{YX}), 11171199$ (ST), 11141201 (ST)), and by the Fundamental Research Funds for the Central Universities (08143042 (YX) and by the International Development Research Center, Ottawa, Canada (104519-010).

\section{References}

Arcietro, J. C., Jackson, T. L. \& Kirschner, D. E. [2004] "A mathematical model of tumor-immune evasion and siRNA treatment," Discrete Cont. Dyn. Syst.-B 4(1), 39-58.

Bonotto, E. M. [2009] "LaSalle's theorems in impulsive semidynamical system," Nonlinear Anal. TMA 71, 2291-2297.

Bonotto, E. M. \& Federson, M. [2008] "Limit sets and the Poincaré Bendixson theorem in impulsive semidynamical systems," J. Differ. Equ. 244,2334-2349.

Borges, F. S., Iarosz, K. C., Ren, H. P., et al. [2014] "Model for tumour growth with treatment by continuous and pulsed chemotherapy," Biosystem 116, 43-48.

Corless, R. M., Connet, G. H., Hâre, D. E. G., Jeffrey, D. J. \& Knuth, D. E. [1996] "On the Lambert W function," Adv. Comput. Math. 5, 329-359.

De Angelis, E., Marasco, A. \& Romano, A. [2003] "Bifurcation analysis for mean field modelling of tumor and immune system competition," Math. Comput. Model. 37, 1131-1142.

De Pillis, L. G. \& Radunskaya, A. [2003] "The dynamics of an optimally controlled tumor model: a case study," Math. Comput. Model. 37, 1221-1244.

Duffey, B. G., Choyke, P. L., Clenn, G., Grubb, R. L., Venzon, D., Linehan, W. M. \& Walther, M. M. [2004] "The relationship between renal tumor size and metastases in patients with von Hippel-Lindau disease," J. Urol. 172, 63-65.

Foryś, U. \& Bodbar, M. [2003] "Time delays in regulatory apoptosis for solid avascular tumor," Math. Comput. Model. 37, 1211-1220.

Gubin, M. M., Zhang, X. L., Schuster, H., et al. [2014] "Checkpoint blockade cancer immunotherapy targets tumour-specific mutant antigens," Nature 545, 577-593.

Hegmans, J. P., Hemmes, A., Aerts, J. G., Hoogsteden, H. C. \& Lambrecht B. N. [2005] "Immunotherapy of murine malignant mesothelioma using tumor lysate-pulsed dendritic cells," Am. J. Respir. Crit. Care. Med. 171(10), 1168-1177.

Herring, J. C., Enquist, E. G., Chernoff, A., Linehan, W. M., Choyke, P. L. \& Walther, P. L. [2001] "Parenchymal sparing surgery in patients with hereditary renal cell carcinoma: 10-year experience," J. Urol. 165, 777-781. 
Janeway, C. A., Travers, J. P., Walport, M. \& Shlomchik, M. J. [2001] Immunobiology: The Immune system in health and disease, 5th edition (Garland Publishing, New York).

Karen, M. P. \& Jonathan, W. U. [2005] "Mathematical models of cancer dormancy," Leukemia \& Lymphoma 46(3), 313-327.

Kaul, S. K. [1990] "On impulsive semidynamical systems," J. Math. Anal. Appl. 150, 120-128.

Kuznetsov, V. A. \& Knott, G. D. [2001] "Modeling tumor regrowth and immunotherapy," Math. Comput. Model. 33, 1275-1287.

Kuznetsov, V. A., Makalkin, I. A., Taylor, M. A. \& Petelson, A. S. [1994] "Nonlinear dynamics of immunogenic tumors: parameter estimation and global bifurcation analysis," Bull. Math. Biol. 56(2), 295-321.

Lefever, R., Hiernaux, J., Urbain, J. \& Meyers, P. [1992] "On the kinetics and optimal specificity of cytotoxic reactions mediated by T-lymphocyte clones," Bull. Math. Biol. 54, 839-873.

Liang, J. H., Tang, S. Y., Nieto, J. J., \& Cheke, R. A. [2013] "Analytical methods for detecting pesticide switches with evolution of pesticide resistance," Math. Biosci. 245, 249-257.

Look, A .T., Schriber, T. J., Nawrochi, J. F. \& Murphy, W. H. [1981] "Computer simulation of the cellular immune response to malignant lymphoid cells:Logic of approach, model design and laboratory verification," Immunol. 43, 677-690.

Lou, L., Lou, Y. L. \& Wu, J. H. [2012] "Threshold virus dynamics withimpulsive antiretroviral drug effects," J. Math. Biol. 65, 623-652.

Nie, L. F., Teng, Z. D. \& Guo, B. Z. [2013] "A state dependent pulse control strategy for a SIRS epidemic system," Bull. Math. Biol. 75, 1697-1715.

Powles, T., Eder, J. P., Fine, G. D., et al. [2014] "MPDL3280A (anti-PD-L1) treatment leads to clinical activity in metastatic bladder cancer," Nature 515, 558-569.

Qian, L. N., Lu, Q. S., Meng, Q. G. \& Feng, Z. S. [2010] "Dyanmical behaviors of a prey-predator system with impulsive control," J. Math. Anal. Appl. 363, 345-356.

Salleem, M. \& Angrawal, T. [2012] "Complex dynamics in a mathematical model of tumor growth with time delays in the cell proliferation," Internationat Journal of Scientific and Research 2, 1-7.

Schmiegel, W., Schmielau, J., Henne-Bruns, D., et al. \{1997] "Cytokine-mediated enhancement of epidermal growth factor receptor expression provides an immunological approach to the therapy of pancreatic cancer," Proc. Natl. Acad. Sci. USA 94, 12622-12626.

Shiao, S. L., Canesan, A. P., Rugo, H. S. \& Coussens, L. M. [2011] "Immune microenviroments in solid tumors: new targets for therapy," Genes. Dev. 25, 2559-2572.

Shulgin, B., Stone, L. \& Agur, Z. [1998] "Pulse vaccination strategy in the SIR epedemic model," Bull. Math. Biol. 60, 1123-1148.

Šímová J., Indrová, M., Bieblová, J., et al. [2010] "Therapy for minimal residual tumor disease: $\beta$ galactosylceramide inhibits the growth of recurrent HPV16-associated neoplasms after surgery and chemotherapy," Int. J. Cancer 126, 2997-3004.

Steve, B., Andrew, C., et al. [2005] "Partial, but not complete, tumor-debulking surgery promotes protective antitumor memory when combined with chemotherapy and adjuvant immunotherapy," Cancer Res. 65, 7580-7584.

Subuyanto, Mamat M, Mohammad F. A., et al. [2014] "Non-dimensional system for analysis equilibrium point mathematical model of tumor growth," Appl. Math. Sci. 8(2), 91-98.

Sunay, M. E., Marincola, F., Khleif, S. N., et al. [2013] "Forcus on the target: the tumor microenvironment, Society for Immunotherapy of Cancer Annual Meeting Workshop, October 24th-25th 2012," $J$. Immunother. Cancer 1-9.

Tang, S. Y. \& Cheke, R. A. [2005] "State-dependent impulsive models of integrated pest management (IPM) strategies and their dynamic consequences," J. Math. Biol. 50, 227-240.

Tang, S. Y. \& Cheke, R. A. [2008] "Models for integrated pest control and their biological implications," Math. Biosci. 215, 115-125.

Tang, S. Y., Liang, J. H., Tan, Y. \& Cheke, R. A. [2013] "Threshold conditions for integrated pest management models with pesticides that have residual effects," J. Math. Biol. 66, 1-35.

Tang, S. Y. \& Xiao, Y. N. [2007] "One-compartment model with Michaelis-Menten elimination kinetics 
and therapeutic window: an analytical approach," J. Pharmacokin. Biopharm. 34, 807-827.

Touboul, J. \& Brette, R. [2009] "Spiking dynamics of bidimensional integrate-and-fire neurons," SIAM J. Appl. Dyn. Syst. 8(4), 1462-1506.

Walther, M. M., Choyke, P. L., Glenn, G., Lyne, J. C., Rayford, W., Venzon, D. \& Linehan, W. M. [1999] "Renal cancer in families with hereditary renal cancer: Prospective analysis of a tumor size threshold for renal parenchymal sparing surgery," J. Urol. 161(5), 1475-1479.

Wilson, S. \& Levy, D. [2012] "A mathemetical model of the enhancement of tumor vaccine efficacy by immunotherapy," Bull. Math. Biol. 74, 1485-1500.

Xiao, Y. N., Miao, H. Y., Tang, S. Y. \& Wu, H.L. [2013] "Modeling antiretroviral drug responses for HIV-1 infected patients using differential equation models," Adv. Drug Deliv. Rev. 65, 940-953.

Yamaguchi, S., Ogiue-Ikeda, M., Sekino, M. \& Ueno, S. [2006] "Effects of Pulsed Magnetic Stimulation on Tumor Development and Immune Functions in Mice," Bioelectromagnetics 27, 64-72.

Yang, Y. P. \& Xiao, Y. N. [2012] "Threshold dynamics for compartmental epidemic models with impulses," Nonlinear Anal. RWA 13, 224-234.

Yang, Y., Xiao, Y. \& Wu, J. [2013] "Pulse HIV Vaccination: Feasibility for Virus Eradication and Optimal Vaccination Schedule," Bull. Math. Biol. 75(5), 725-751.

Zeng, G. Z., Chen, L. S. \& Sun, I. H. [2006] "Existence of periodic solution of order of one planar impulsive autonomous system," J. Comp. Appl. Math. 186, 466-481.

\section{Appendix A}

Definition 3. The Lambert W function [Corless et al., 1996] is defined to be a multivalued inverse of the function $z \mapsto z e^{z}$ satisfying

$$
\text { LambertW }(z) \exp (\operatorname{Lambert} W(z))=z \text {. }
$$

And we denote it as $W$ for simplicity. Note that if $z>-1$ then the function $z \exp (z)$ has the positive derivative $(z+1) \exp (z)$. Define the inverse function of $z \exp (z)$ restricted on the interval $[-1,+\infty)$ to be $W(0, z)$. Similarly, we define the inverse function of $z \exp (z)$ restricted on the interval $(-\infty,-1]$ to be $W(-1, z)$. The branch $W(0, z)$ is defined on the interval $\left[-e^{-1},+\infty\right)$ and it is a monotonically increasing function with respect to $z$. And the branch $W(-1, z)$ is defined on the interval $\left[-e^{-1}, 0\right)$ and it is a monotonically decreasing function with respect to $z$. 

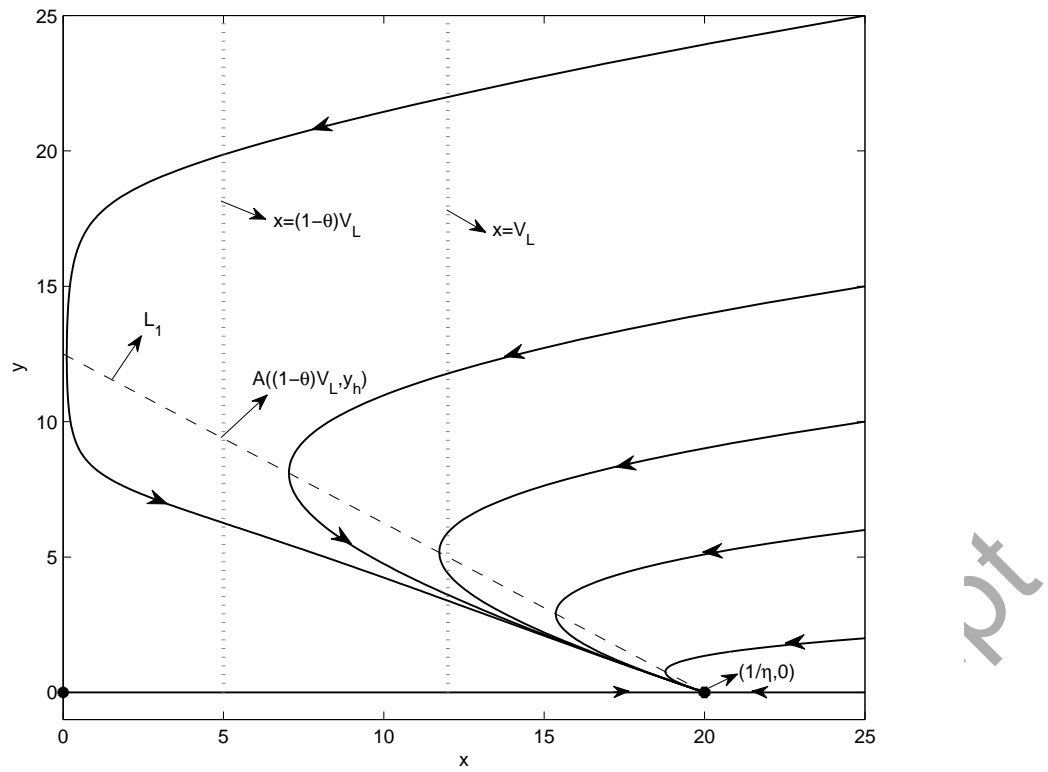

Fig. 1. The topological trajectory map of system (1) when there is no positive equilibrium.

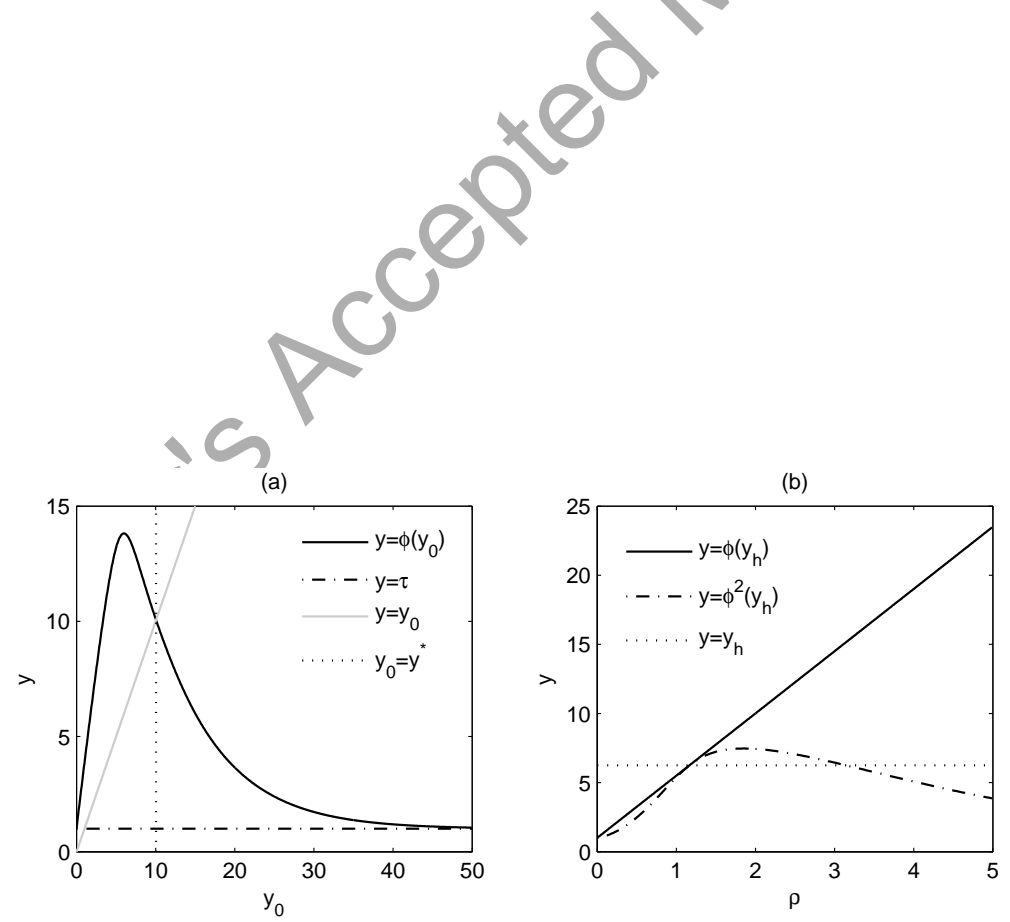

Fig. 2. (a) The Poincaré map; (b) The curves of $\phi\left(y_{h}\right)$ and $\phi^{2}\left(y_{h}\right)$ as $\rho$ increases while $\eta=0$. 

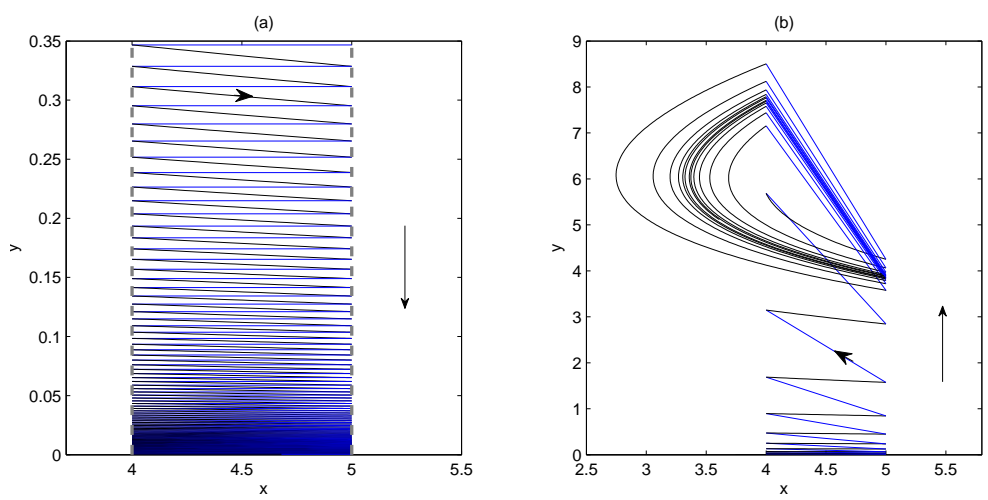

Fig. 3. (a) Solution trajectory of system (2) when $\rho=1$ with $\left|\mu_{2}\right| \leq 1$; (b) Solution trajectory of system 2 when $\rho=2$ with $\left|\mu_{2}\right| \geq 1$. Other parameter values are: $r=5, \eta=0.01, p=0.8, c=0.3, \omega=1, q=0.23, \delta=0.3, \theta=0.2, \tau=0$.
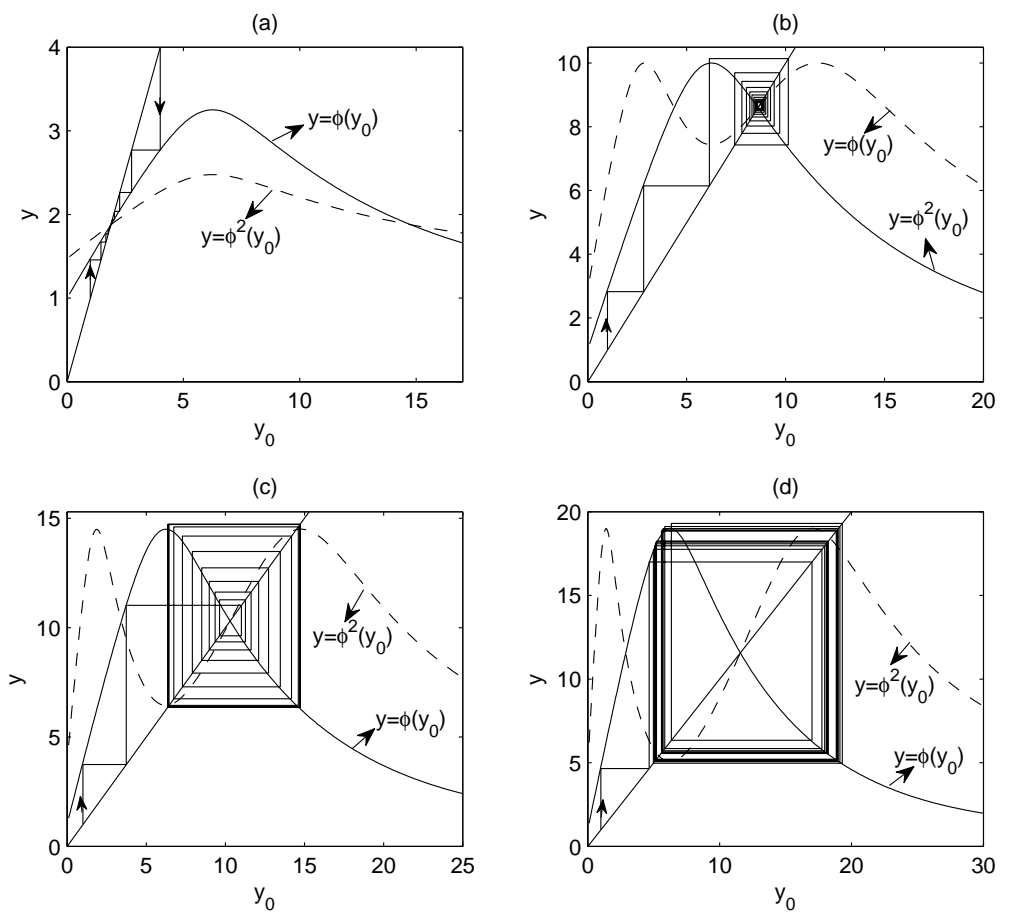

Fig. 4. The Poincaré map $\phi\left(y_{0}\right)$ and $\phi^{2}\left(y_{0}\right)$ by choosing different values of $\rho$ with (a) $\rho=0.5$; (b) $\rho=2 ;$ (c) $\rho=3$; (d) $\rho=4$. The existence of an order-1 periodic solution has been shown in (a) and (b) while (c) and (d) show the existence of order-2 and order-4 periodic solutions, respectively. 


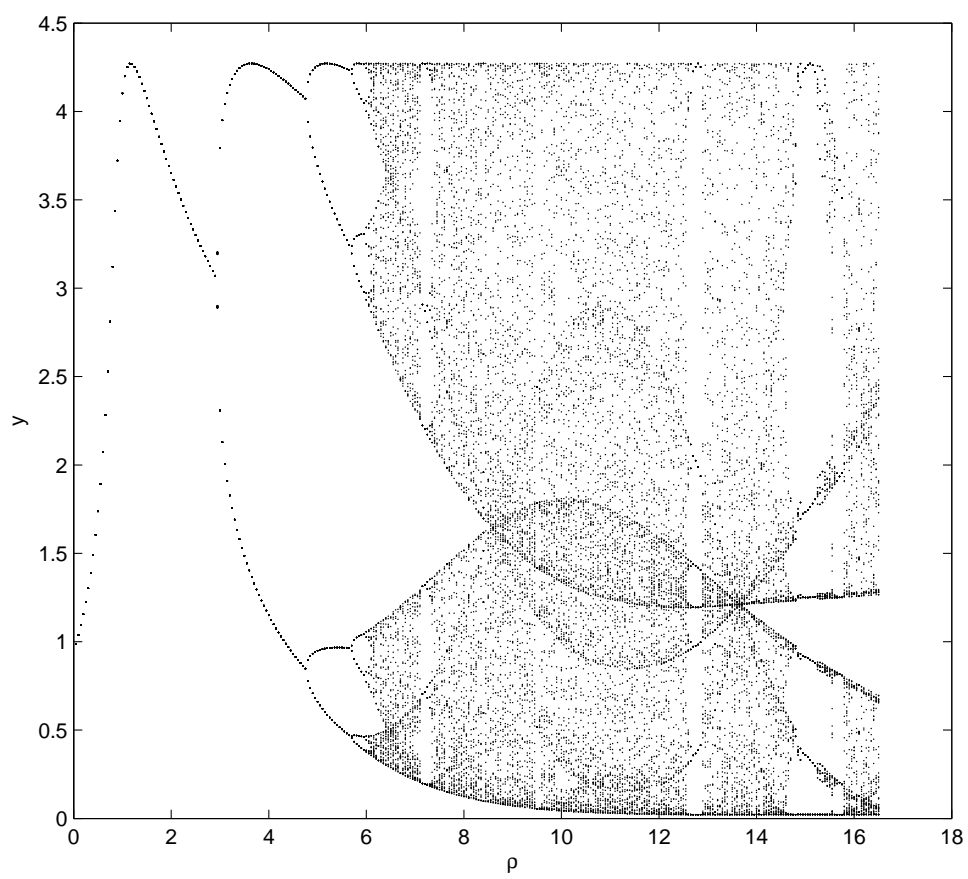

Fig. 5. Bifurcation diagram of positive order-k periodic solution to $\rho$ with $r=5, \eta=0.01, p=0.8, c=0.3, \omega=1, q=$ $0.23, \delta=0.3, V_{L}=5, \tau=1, \theta=0.2$.
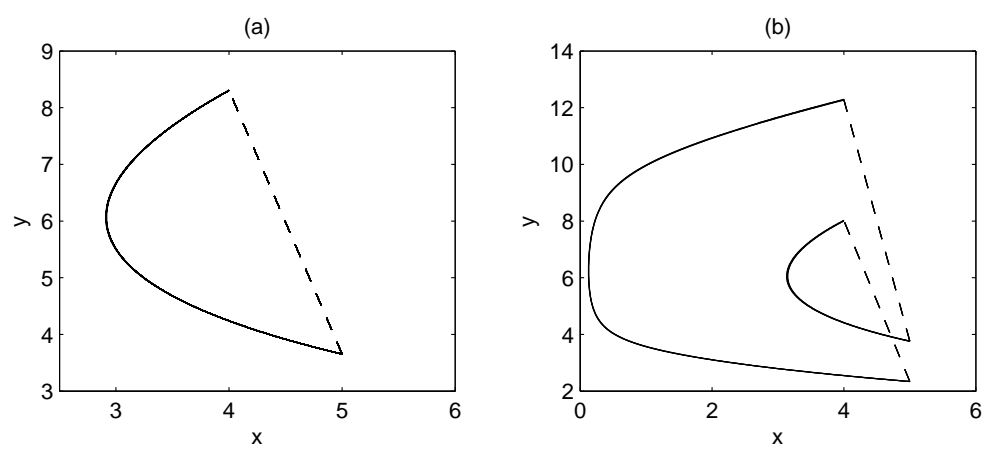

(c)
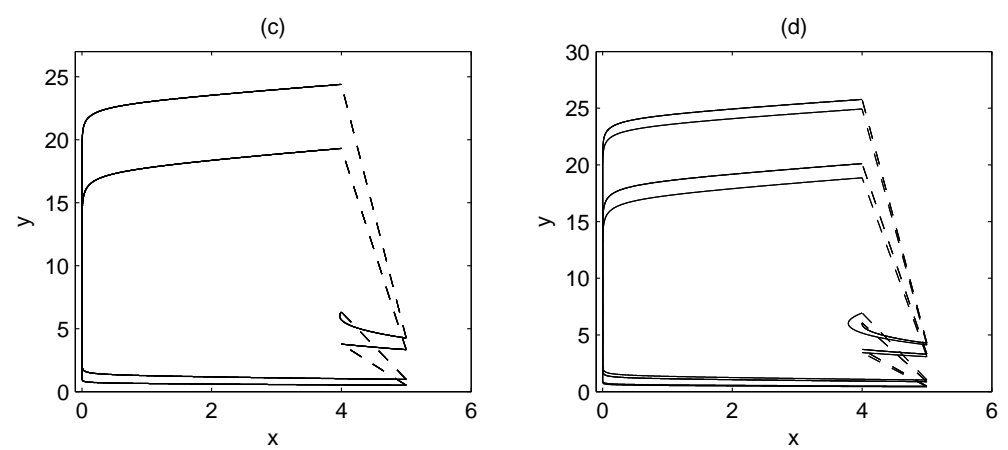

Fig. 6. (a) Order-1 periodic solution with $\rho=2$; (b) Order-2 periodic solution with $\rho=3$; (c) Order-4 periodic solution with $\rho=5.5$; (d) Order-8 periodic solution with $\rho=5.8$. All the other parameter values are: $r=5, \eta=0.01, p=0.8, c=0.3, \omega=$ $1, q=0.23, \delta=0.3, V_{L}=5, \tau=1, \theta=0.2$. 

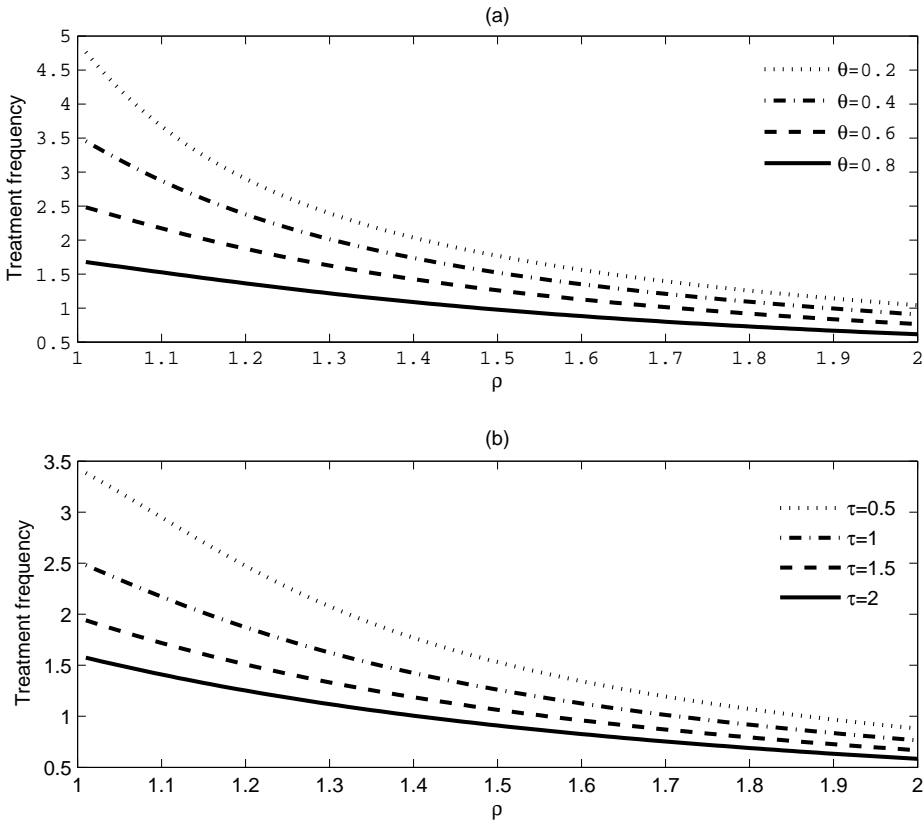

Fig. 7. Variation in treatment frequency with the growth rate of effector cells $\rho$. (a) $\tau=1$; (b) $\theta=0.6$.
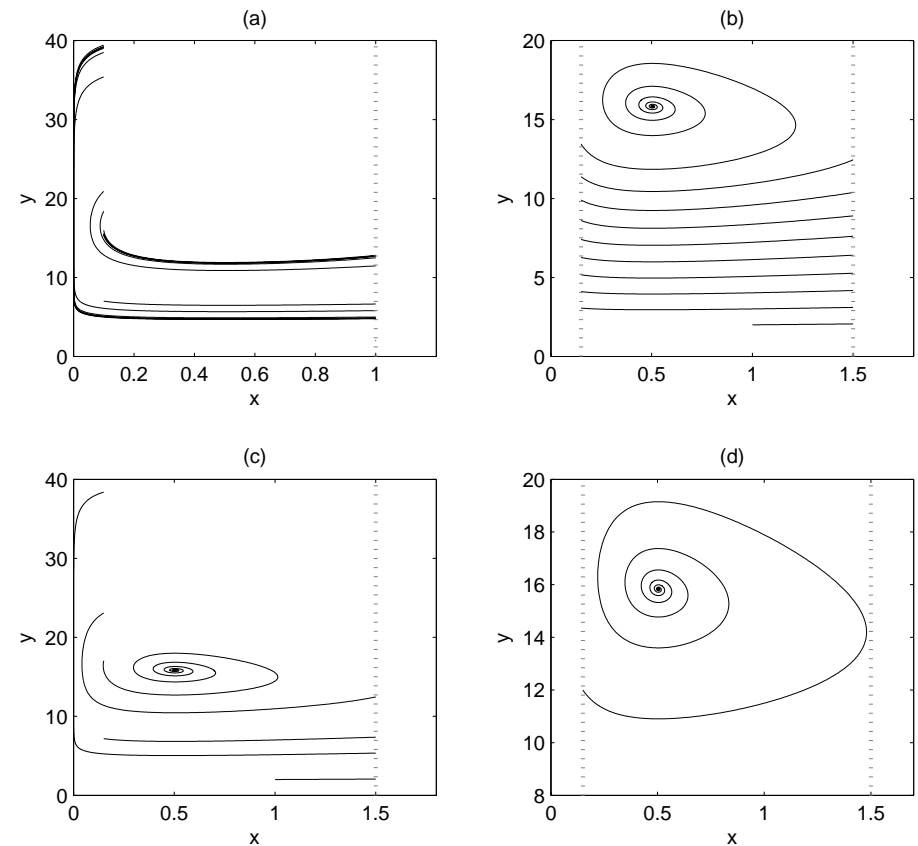

Fig. 8. Trajectory solution of system (2) when two positive equilibria of system (1) are feasible. (a) $V_{L}=1, \rho=3$ with the initial value $(x(0), y(0))=(1,2)$; (b) $V_{L}=1.5, \rho=1$ with the initial value $(x(0), y(0))=(1,2) ;(\mathrm{c}) V_{L}=1.5, \rho=3$ with the initial value $(x(0), y(0))=(1,2) ;(\mathrm{d}) V_{L}=1.5, \rho=3$ with the initial value $(x(0), y(0))=(1.15,12)$. The other parameter values are: $r=5, p=0.3, \omega=0.3, c=0.95, q=0.23, \delta=0.3, \eta=0.1, \tau=1, \theta=0.9$. 